\title{
Analysis of nested multilevel Monte Carlo using approximate Normal random variables
}

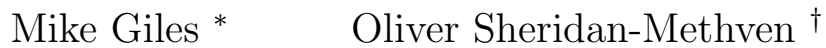

February 17, 2021

\begin{abstract}
The multilevel Monte Carlo (MLMC) method has been used for a wide variety of stochastic applications. In this paper we consider its use in situations in which input random variables can be replaced by similar approximate random variables which can be computed much more cheaply. A nested MLMC approach is adopted in which a twolevel treatment of the approximated random variables is embedded within a standard MLMC application. We analyse the resulting nested MLMC variance in the specific context of an SDE discretisation in which Normal random variables can be replaced by approximately Normal random variables, and provide numerical results to support the analysis.
\end{abstract}

\section{Introduction}

Following the initial work of Heinrich [1] on parametric integration and Giles [2] on stochastic differential equations (SDEs), there has been huge development in the application of multilevel Monte Carlo (MLMC) methods to a wide variety of stochastic modelling contexts. This includes partial differential equations (PDEs) with stochastic coefficients [3, 4], stochastic PDEs [5], continuous-time Markov process models of biochemical reactions [6, 7], Markov Chain Monte Carlo [8, 9], nested simulation [10, 11], probability density estimation $[12,13]$, and reliability estimation $[14,15]$. A review of research on MLMC is provided by Giles [16].

In this paper we are concerned with the development and analysis of a new class of MLMC methods involving approximate probability distributions. Most numerical methods for simulating stochastic models start from random inputs from a variety of well-known distributions: Normal, Poisson, binomial, non-central $\chi^{2}$, etc. Generating samples which have a distribution which matches the desired distribution to within the limits of finite precision arithmetic can be a significant part of the overall computational cost of the simulation. Here we consider what can be achieved if it is also possible to generate approximate random variables (random variables with a distribution which is only approximately correct) at a greatly reduced cost. We will show that a nested MLMC approach can be adopted in which a two-level treatment of the approximated random variables is embedded within a standard MLMC application. We then analyse the MLMC variance of the resulting treatment in the

*mike.giles@maths.ox.ac.uk

†oliver.sheridan-methven@hotmail.co.uk 
specific context of an SDE discretisation in which Normal random variables can be replaced by approximately Normal random variables.

The most relevant prior research is the work of Giles, Hefter, Mayer and Ritter [17, 18]. This research was in the context of Information Based Complexity (IBC), working with a complexity model which counted the number of individual random bits, rather than viewing each standard uniformly-distributed random variable as having a unit cost. Fundamental to the algorithms in these papers was the use of quantised Normal random variables, which is the first of the three approximations to be discussed in the next section. Some elements of the analysis in this paper build on the ideas and analysis in those papers, but the context is quite different in aiming to minimise the real-world execution cost of MLMC algorithms on modern CPUs and GPUs, and the specifics of the proposed method are quite different in using a nested MLMC approach.

Another relevant paper is by Müller et al. [19]. In this work they use three- and fourpoint approximations to the Normal distribution, equivalent to a piecewise constant approximation of $\Phi^{-1}(U)$ on 3 or 4 intervals of a non-uniform size, chosen so that the leading moments are the same as for the standard Normal distribution. The way in which these are used within the MLMC construction violates, to a small extent, the usual telescoping summation which lies at the heart of the MLMC method, and therefore they have to be careful to bound the magnitude of this error. This new error is related to the fact that if $Z_{1}$ and $Z_{2}$ are unit Normal random variables, then so too is their sum $\left(Z_{1}+Z_{2}\right) / \sqrt{2}$; in an SDE application, this is important in MLMC so that the sum of two Brownian increments from timesteps of size $h$ corresponds to a Brownian increment from a timestep of size $2 h$. However, this is no longer true when using approximate Normal distributions; the sum $\left(\widetilde{Z}_{1}+\widetilde{Z}_{2}\right) / \sqrt{2}$ is still an approximation of a unit Normal random number, but in general it does not come from exactly the same distribution as $\widetilde{Z}_{1}$ and $\widetilde{Z}_{2}$.

Similar ideas have also been investigated by Belomestny and Nagapetyan [20] who avoid errors in the telescoping summation by using different approximate distributions on each level of MLMC refinement. However, in the present paper we prefer to avoid these difficulties entirely by using the same approximate distribution throughout within a nested MLMC treatment; we think this will generalise better to different approximations well-suited to different computer hardware, and to applications with more complex distributions. We also consider three different kinds of approximations, some of which will generalise better to distributions such as the non-central $\chi^{2}$-distribution in which there are additional parameters which may vary for each random number sample; in such cases a lookup table based on quantisation could become unreasonably large.

\section{Approximate Normal distributions}

There are many ways in which approximate Normal variables can be generated. In this paper we consider three methods, each of which can be viewed as an approximation of the generation of Normal random variables through the inversion of the Normal CDF function, $Z=\Phi^{-1}(U)$, with $U$ being a uniform random variable on the unit interval $(0,1)$. The corresponding approximations all have the form $\widetilde{Z}=\widetilde{Q}(U)$, so that it is possible to generate coupled pairs $(Z, \widetilde{Z})$ from the same random input $U$.

The three approximations are all motivated by the different hardware features of modern CPUs and GPUs (manycore graphics processing units). Their analysis, implementation 

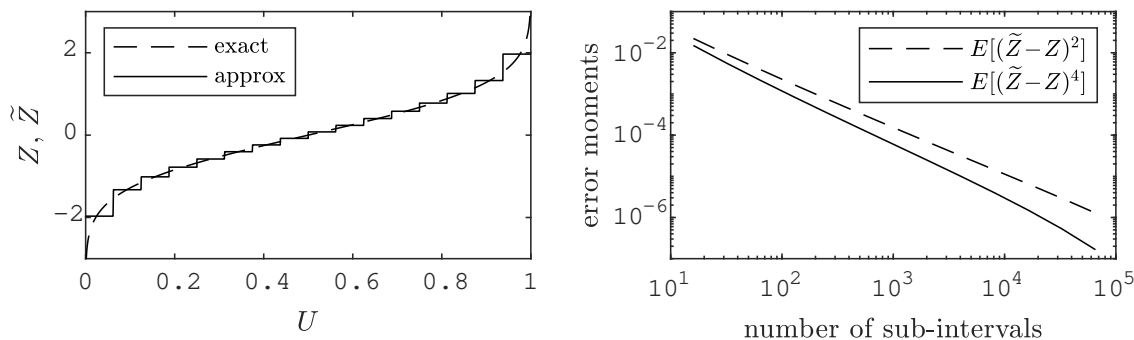

(a) Quantised approximation
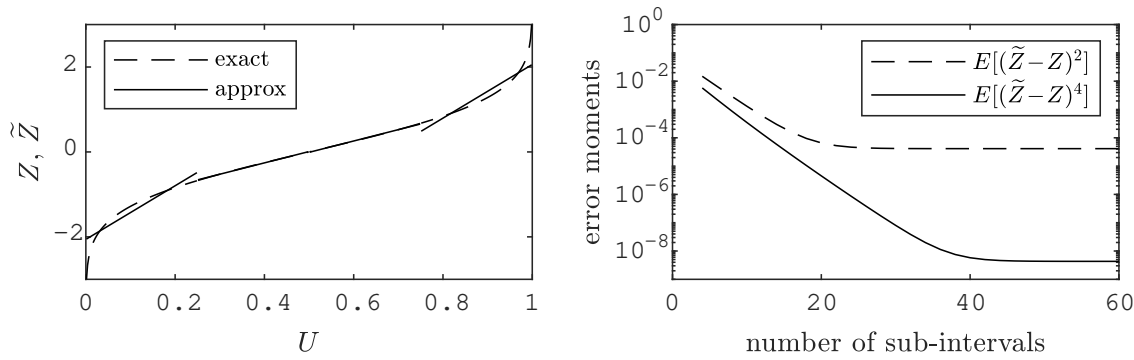

(b) Piecewise linear approximation on dyadic intervals


(c) Polynomial approximation

Figure 1: Three approximations of the inverse Normal CDF.

details, and resulting execution performance are explained more fully in [21, 22].

Note that we are not concerned with the computational cost of generating the uniform random numbers $U$. This is because we can follow Giles et al. [18] in using a trick due to Bakhvalov [23] to very efficiently generate a set of uniformly-distributed values $\left\{U_{1}, U_{2}, \ldots\right\}$ with pairwise independence (i.e. for any two indices $i \neq j, U_{i}$ is independent of $U_{j}$ ) at a cost of less than 3 computer operations, on average. In essence, the procedure is very similar to the digital shift used to transform Sobol' points in a randomised Quasi Monte Carlo computation [24].

\subsection{Quantised approximation}

The first approximation is a simple piecewise constant approximation on $K=2^{q}$ intervals of size $2^{-q}$, with the value $\widetilde{Q}(U)$ on the $k$-th interval $I_{k}=\left[(k-1) 2^{-q}, k 2^{-q}\right]$, given either by the average value of $\Phi^{-1}(U)$ on the interval, or alternatively the value at the mid-point.

The index $k$ corresponds to the first $q$ bits of the binary expansion for $U$, and this quantised approximation is the one considered by Giles et al. [17, 18] since each random variable $\widetilde{Z}$ can be generated based directly on $q$ random bits, each independently taking 
the value 0 or 1 with equal probability. Extending their analysis, it can be proved that

$$
\mathbb{E}\left[|\widetilde{Z}-Z|^{p}\right]=o\left(2^{-q}\right) .
$$

Figure $1\left(\right.$ a) illustrates the approximation for $K=16$, and also has plots of $\mathbb{E}\left[(\widetilde{Z}-Z)^{2}\right]$ and $\mathbb{E}\left[(\widetilde{Z}-Z)^{4}\right]$ as a function of $K$, the number of intervals.

For $K=1024$ the mean square error $(\mathrm{MSE})$ is $\mathbb{E}\left[(\widetilde{Z}-Z)^{2}\right] \approx 1.5 \times 10^{-4}$. This size seems a good choice as the lookup table will fit inside the L1 cache of a current generation Intel CPU, leading to a very efficient scalar implementation.

\subsection{Piecewise linear approximation on dyadic intervals}

The second approximation uses a discontinuous piecewise linear approximation on a geometric sequence of sub-intervals. To be specific, for a given ratio $\frac{1}{2} \leq r<1, K$ sub-intervals on $\left[0, \frac{1}{2}\right]$ are defined by

$$
I_{k}=\left[\frac{1}{2} r^{k}, \frac{1}{2} r^{k-1}\right], \quad k=1,2, \ldots K-1, \quad I_{K}=\left[0, \frac{1}{2} r^{K-1}\right] .
$$

In the particular case $r=\frac{1}{2}$, given an input $0<U<\frac{1}{2}$, the corresponding sub-interval index $k$ can be determined by computing the integer part of $\log _{2} U$, which can be implemented very efficiently due to the floating point format of real numbers.

On each sub-interval $I_{k}, \widetilde{Q}(U)$ is defined as the least-squares linear best fit approximation to $\Phi^{-1}(U)$, and the approximation on $\left[\frac{1}{2}, 1\right]$ is defined by $\widetilde{Q}(U)=-\widetilde{Q}(1-U)$. Standard analysis of the accuracy of piecewise linear interpolation leads to the result that

$$
\mathbb{E}\left[|\widetilde{Z}-Z|^{p}\right]=o\left(r^{K}\right)+O\left((1-r)^{2 p}\right),
$$

where the first term comes from the two end intervals $\left[0, \frac{1}{2} r^{K-1}\right]$ and $\left[1-\frac{1}{2} r^{K-1}, 1\right]$, and the second term comes from the other intervals. Note that to achieve convergence to zero requires that both $r \rightarrow 1$ and $r^{K} \rightarrow 0$.

Figure 1(b) illustrates the approximation for $r=\frac{1}{2}, K=2$, and also has plots of $\mathbb{E}\left[(\widetilde{Z}-Z)^{2}\right]$ and $\mathbb{E}\left[(\widetilde{Z}-Z)^{4}\right]$ as a function of $2 K$, the number of intervals, with fixed $r=\frac{1}{2}$. Note that for both $\mathbb{E}\left[(\widetilde{Z}-Z)^{2}\right]$ and $\mathbb{E}\left[(\widetilde{Z}-Z)^{4}\right]$ the $(1-r)^{2 p}$ error term eventually dominates once $r^{K}$ is sufficiently small.

For $K=16$ the MSE is $\mathbb{E}\left[(\widetilde{Z}-Z)^{2}\right] \approx 4 \times 10^{-5}$. This size is significant because in single precision it is possible to perform the table lookup within a single 512-bit AVX vector register on current generation Intel Xeon CPUs, giving a very efficient vector implementation.

\subsection{Polynomial approximation}

The final method is a simple polynomial approximation

$$
\widetilde{Q}(U) \equiv \sum_{k=1}^{K} a_{k}\left(U-\frac{1}{2}\right)^{2 k-1},
$$

with the coefficients $a_{k}$ determined by a least squares best fit to $\Phi^{-1}(U)$. This method is particularly efficient on GPUs as it avoids the need for a table lookup, however it is also the least accurate of the three approximations for realistic polynomial sizes. 
Figure $1(\mathrm{c})$ illustrates the approximation for $K=2$, and also has plots of $\mathbb{E}\left[(\widetilde{Z}-Z)^{2}\right]$ and $\mathbb{E}\left[(\widetilde{Z}-Z)^{4}\right]$ as a function of $2 K-1$, the degree of the polynomial.

For $K=4$ the MSE is $\mathbb{E}\left[(\widetilde{Z}-Z)^{2}\right] \approx 2.6 \times 10^{-3}$; this seems a good balance between computational cost and accuracy, and will be used in the numerical experiments later.

\section{MLMC algorithms}

In this section we begin with a quick recap of the multilevel Monte Carlo method, and then discuss how a nested version of MLMC can be used with approximate distributions. The third part then applies the ideas to the simulation of SDE solutions, using approximate Normal random variables.

\subsection{Standard MLMC}

If $P$ is a random variable which is a function of a set of random inputs $\omega$, then the Monte Carlo estimate for the expected value $\mathbb{E}[P]$ is the simple average

$$
N^{-1} \sum_{n=1}^{N} P\left(\omega^{(n)}\right)
$$

where the $\omega^{(n)}, n=1,2,3, \ldots, N$ are i.i.d. samples of $\omega$. To achieve a root-mean-square (RMS) error of $\varepsilon$ requires $N \approx \varepsilon^{-2} V$ samples, where $V=\mathbb{V}[P]$ is the variance. If each sample costs $C$ then the total cost is approximately $\varepsilon^{-2} V C$.

Suppose now that $\widetilde{P} \approx P$, then since $\mathbb{E}[P]=\mathbb{E}[\widetilde{P}]+\mathbb{E}[P-\widetilde{P}]$ we can instead use the estimator

$$
N_{0}^{-1} \sum_{n=1}^{N_{0}} \widetilde{P}\left(\omega^{(0, n)}\right)+N_{1}^{-1} \sum_{n=1}^{N_{1}}\left(P\left(\omega^{(1, n)}\right)-\widetilde{P}\left(\omega^{(1, n)}\right)\right) .
$$

The cost of this estimator is $N_{0} C_{0}+N_{1} C_{1}$, and the variance is $V_{0} / N_{0}+V_{1} / N_{1}$, where $V_{0} \equiv \mathbb{V}[\widetilde{P}], V_{1} \equiv \mathbb{V}[P-\widetilde{P}]$, if all of the $\omega^{(\ell, n)}$ are independent. Minimising the cost subject to the same accuracy requirement gives the total cost $\varepsilon^{-2}\left(\sqrt{V_{0} C_{0}}+\sqrt{V_{1} C_{1}}\right)^{2}$. which is significantly less than $\varepsilon^{-2} V C$ if $C_{0} \ll C$ and $V_{1} \ll V$.

To give a quantitative example, suppose that $C_{0}=10^{-1} C, C_{1}=C$, so the cost of evaluating $\widetilde{P}$ is 10 times less than evaluating $P$, and $V_{0}=V, V_{1}=10^{-3} V$. In that case, the total cost is approximately $0.12 \varepsilon^{-2} V C$, a factor of 8 savings compared to the original Monte Carlo calculation.

This two-level calculation is easily generalised to a multilevel treatment. Given a sequence of increasingly accurate (and costly) approximations $\widehat{P}_{0}, \widehat{P}_{1}, \widehat{P}_{2}, \ldots \longrightarrow P$, for example from the approximation of an SDE using $2^{\ell}$ timesteps on level $\ell$, then

$$
\mathbb{E}\left[\widehat{P}_{L}\right]=\mathbb{E}\left[\widehat{P}_{0}\right]+\sum_{\ell=1}^{L} \mathbb{E}\left[\widehat{P}_{\ell}-\widehat{P}_{\ell-1}\right]
$$

and so the MLMC estimate for $\mathbb{E}\left[\widehat{P}_{L}\right]$ is

$$
\widehat{Y} \equiv N_{0}^{-1} \sum_{n=1}^{N_{0}} \widehat{P}_{0}^{(n)}+\sum_{\ell=1}^{L} N_{\ell}^{-1} \sum_{n=1}^{N_{\ell}}\left(\widehat{P}_{\ell}^{(\ell, n)}-\widehat{P}_{\ell-1}^{(\ell, n)}\right) .
$$


The expected value of the estimator is $\mathbb{E}\left[\widehat{P}_{L}\right]$, and the MSE can be decomposed into the sum of the variance and the square of the bias to give

$$
\mathrm{MSE}=\mathbb{V}[\widehat{Y}]+\left(\mathbb{E}\left[\widehat{P}_{L}-P\right]\right)^{2}=\sum_{\ell=0}^{L} N_{\ell}^{-1} V_{\ell}+\left(\mathbb{E}\left[\widehat{P}_{L}-P\right]\right)^{2}
$$

where $V_{0} \equiv \mathbb{V}\left[\widehat{P}_{0}\right]$, and for $\ell \geq 1, V_{\ell} \equiv \mathbb{V}\left[\widehat{P}_{\ell}-\widehat{P}_{\ell-1}\right]$.

If level $L$ is chosen so that $\left|\mathbb{E}\left[\widehat{P}_{L}-P\right]\right|<\varepsilon / \sqrt{2}$, then an overall RMS error of $\varepsilon$ can be achieved at a total cost of approximately

$$
2 \varepsilon^{-2}\left(\sum_{\ell=0}^{L} \sqrt{V_{\ell} C_{\ell}}\right)^{2}
$$

where $C_{\ell}$ is the cost of a single sample of $\widehat{P}_{\ell}-\widehat{P}_{\ell-1}$. If the product $V_{\ell} C_{\ell}$ decreases exponentially with level then the overall cost is $O\left(\varepsilon^{-2}\right)$, corresponding to an $O(1)$ cost per sample on average, much less than for the standard Monte Carlo method. For further details see $[2,16]$.

\subsection{Nested MLMC for approximate distributions}

When using random variables from approximate distributions, the first possibility is to use the two-level treatment discussed before, with

$$
\mathbb{E}[P]=\mathbb{E}[\widetilde{P}]+\mathbb{E}[P-\widetilde{P}]
$$

In this case, each sample $P-\widetilde{P}$ would use the same underlying stochastic sample for both $P$ and $\widetilde{P}$, for example using the same input uniform random variable $U$, and then applying the inverse of either the true CDF or an approximate CDF to produce the random variables required to compute $P$ and $\widetilde{P}$, respectively. As stated before, this can give considerable savings if the cost of computing $\widetilde{P}$ is much less than the cost of computing $P$, and $\mathbb{V}[P-\widetilde{P}] \ll$ $\mathbb{V}[P]$.

However, what can we do if our starting point is an MLMC expansion in some other quantity, such as the timestep? In that case we can use nested MLMC, an idea discussed by Giles, Kuo and Sloan [25] which is a generalisation of Multi-Index Monte Carlo by Haji-Ali, Nobile and Tempone [26]. We start from the usual MLMC expansion and then split each of the required expectations into two pieces, one using the approximate random variables and the other computing the required correction,

$$
\begin{aligned}
\mathbb{E}\left[\widehat{P}_{L}\right]= & \mathbb{E}\left[\widehat{P}_{0}\right]+\sum_{\ell=1}^{L} \mathbb{E}\left[\widehat{P}_{\ell}-\widehat{P}_{\ell-1}\right] \\
= & \mathbb{E}\left[\widetilde{P}_{0}\right]+\mathbb{E}\left[\widehat{P}_{0}-\widetilde{P}_{0}\right] \\
& +\sum_{\ell=1}^{L}\left\{\mathbb{E}\left[\widetilde{P}_{\ell}-\widetilde{P}_{\ell-1}\right]+\mathbb{E}\left[\left(\widehat{P}_{\ell}-\widehat{P}_{\ell-1}\right)-\left(\widetilde{P}_{\ell}-\widetilde{P}_{\ell-1}\right)\right]\right\}
\end{aligned}
$$

The pair $\left(\widetilde{P}_{\ell}, \widetilde{P}_{\ell-1}\right)$ are generated in the same way as $\left(P_{\ell}, P_{\ell-1}\right)$, based on the same underlying uniform random variables $U$, but converting them differently into the random variables required for the calculation of $\widehat{P}$ and $\widetilde{P}$. 
As explained in the previous section, the standard MLMC cost to achieve an RMS accuracy of $\varepsilon$ is approximately

$$
C_{\mathrm{MLMC}}=2 \varepsilon^{-2}\left(\sum_{\ell=0}^{L} \sqrt{C_{\ell} V_{\ell}}\right)^{2},
$$

where $C_{\ell}$ is the cost of a single sample of $\widehat{P}_{\ell}-\widehat{P}_{\ell-1}$ on level $\ell$, and $V_{\ell}$ is its variance. The complexity analysis extends naturally to the nested approximate MLMC (AMLMC) algorithm described above, giving a cost of approximately

$$
C_{\mathrm{AMLMC}}=2 \varepsilon^{-2}\left(\sum_{\ell=0}^{L} \sqrt{\widetilde{C}_{\ell} V_{\ell}}+\sqrt{\left(C_{\ell}+\widetilde{C}_{\ell}\right) \widetilde{V}_{\ell}}\right)^{2},
$$

where $\widetilde{C}_{\ell}$ is the cost of one sample of $\widetilde{P}_{\ell}-\widetilde{P}_{\ell-1}$, which has a variance approximately equal to $V_{\ell}$, and $C_{\ell}+\widetilde{C}_{\ell}$ is the cost of one sample of $\left(\widehat{P}_{\ell}-\widehat{P}_{\ell-1}\right)-\left(\widetilde{P}_{\ell}-\widetilde{P}_{\ell-1}\right)$, and $\widetilde{V}_{\ell}$ is its variance.

Note that

$$
\begin{aligned}
C_{\mathrm{AMLMC}} & =2 \varepsilon^{-2}\left(\sum_{\ell=0}^{L} \sqrt{C_{\ell} V_{\ell}} \sqrt{\frac{\widetilde{C}_{\ell}}{C_{\ell}}}\left(1+\sqrt{\left(\frac{C_{\ell}}{\widetilde{C}_{\ell}}+1\right) \frac{\widetilde{V}_{\ell}}{V_{\ell}}}\right)\right)^{2} \\
& \leq C_{\mathrm{MLMC}} \max _{0 \leq \ell \leq L} \frac{\widetilde{C}_{\ell}}{C_{\ell}}\left(1+\sqrt{\left(\frac{C_{\ell}}{\widetilde{C}_{\ell}}+1\right) \frac{\widetilde{V}_{\ell}}{V_{\ell}}}\right)^{2}
\end{aligned}
$$

so that if $\widetilde{V}_{\ell} / V_{\ell} \ll \widetilde{C}_{\ell} / C_{\ell} \ll 1$ then the cost is reduced by a factor of approximately $\max _{\ell} \widetilde{C}_{\ell} / C_{\ell}$.

\subsection{Application to SDE simulation}

To make the ideas in the preceding section more concrete, we consider an application involving the solution of a scalar autonomous SDE,

$$
\mathrm{d} X_{t}=a\left(X_{t}\right) \mathrm{d} t+b\left(X_{t}\right) \mathrm{d} W_{t},
$$

on the time interval $[0, T]$ subject to fixed initial data $X_{0}$. Furthermore, we suppose that we are interested in the expected value of a function of the final path value $\mathbb{E}\left[f\left(X_{T}\right)\right]$.

If $\widehat{X}_{n}$ is an approximation to $X_{n h}$ using a uniform timestep of size $h$, then the simplest numerical approximation is the Euler-Maruyama discretisation,

$$
\widehat{X}_{n+1}=\widehat{X}_{n}+a\left(\widehat{X}_{n}\right) h+b\left(\widehat{X}_{n}\right) \Delta W_{n},
$$

in which the Brownian increment $\Delta W_{n}$ is a Normal random variable with mean 0 and variance $h$, so it can be simulated as $\Delta W_{n} \equiv \sqrt{h} Z_{n}$ where $Z_{n}$ is a unit Normal random variable. In turn $Z_{n}$ can be generated from a uniform $(0,1)$ random variable $U_{n}$ through $Z_{n}=\Phi^{-1}\left(U_{n}\right)$. Using this discretisation the output quantity of interest would be $\widehat{P} \equiv$ $f\left(\widehat{X}_{N}\right)$ where $N=T / h$ is assumed to be an integer.

The simplest way in which we could use approximate Normal random variables would be to keep to a fixed number of timesteps, and generate an approximate output quantity 
$\widetilde{P}$ by replacing the Normals $Z_{n}$ by approximate Normals $\widetilde{Z}_{n} \equiv \widetilde{Q}\left(U_{n}\right)$ generated using the same $U_{n}$.

However, we would like to combine the benefits of MLMC for the time discretisation with the reduced execution cost of approximate Normals and so can instead use the nested MLMC approach. To do this, the key question is how do we compute each sample of $\left(\widehat{P}_{\ell}-\widehat{P}_{\ell-1}\right)-\left(\widetilde{P}_{\ell}-\widetilde{P}_{\ell-1}\right) ?$

Let $h \equiv h_{\ell}$ be the timestep for a fine path on level $\ell$, with $\widehat{X}_{n}^{\mathrm{f}}$ representing the fine path approximation to the SDE solution $X(n h)$ which is computed using the discrete equations

$$
\widehat{X}_{n+1}^{\mathrm{f}}=\widehat{X}_{n}^{\mathrm{f}}+a\left(\widehat{X}_{n}^{\mathrm{f}}\right) h+b\left(\widehat{X}_{n}^{\mathrm{f}}\right) \sqrt{h} Z_{n}
$$

based on the true Normals, $Z_{n}$. The corresponding coarse path approximation $\widehat{X}_{n}^{\mathrm{c}}$ using timesteps of $2 h$ and combined Brownian increments $\Delta W_{n}+\Delta W_{n+1}$ is given by

$$
\begin{aligned}
\widehat{X}_{n+2}^{\mathrm{c}} & =\widehat{X}_{n}^{\mathrm{c}}+2 a\left(\widehat{X}_{n}^{\mathrm{c}}\right) h+b\left(\widehat{X}_{n}^{\mathrm{c}}\right)\left(\Delta W_{n}+\Delta W_{n+1}\right) \\
& =\widehat{X}_{n}^{\mathrm{c}}+2 a\left(\widehat{X}_{n}^{\mathrm{c}}\right) h+b\left(\widehat{X}_{n}^{\mathrm{c}}\right)\left(\sqrt{h} Z_{n}+\sqrt{h} Z_{n+1}\right),
\end{aligned}
$$

for even integers $n$. Alternatively, it is more convenient to write it equivalently as

$$
\widehat{X}_{n+1}^{\mathrm{c}}=\widehat{X}_{n}^{\mathrm{c}}+a\left(\widehat{X}_{\underline{n}}^{\mathrm{c}}\right) h+b\left(\widehat{X}_{\underline{n}}^{\mathrm{c}}\right) \sqrt{h} Z_{n}
$$

where $\underline{n} \equiv 2\lfloor n / 2\rfloor$ is $n$ rounded down to the nearest even number. This gives the same values for $\widehat{X}_{n}^{\mathrm{c}}$ at the even timesteps.

The corresponding discrete equations for the fine and coarse paths computed using the approximate Normal random variables are

$$
\widetilde{X}_{n+1}^{\mathrm{f}}=\widetilde{X}_{n}^{\mathrm{f}}+a\left(\widetilde{X}_{n}^{\mathrm{f}}\right) h+b\left(\widetilde{X}_{n}^{\mathrm{f}}\right) \sqrt{h} \widetilde{Z}_{n}
$$

and

$$
\widetilde{X}_{n+1}^{\mathrm{c}}=\widetilde{X}_{n}^{\mathrm{c}}+a\left(\widetilde{X}_{\underline{n}}^{\mathrm{c}}\right) h+b\left(\widetilde{X}_{\underline{n}}^{\mathrm{c}}\right) \sqrt{h} \widetilde{Z}_{n}
$$

and then finally we obtain

$$
\left(\widehat{P}_{\ell}-\widehat{P}_{\ell-1}\right)-\left(\widetilde{P}_{\ell}-\widetilde{P}_{\ell-1}\right)=\left(f\left(\widehat{X}_{N}^{\mathrm{f}}\right)-f\left(\widehat{X}_{N}^{\mathrm{c}}\right)\right)-\left(f\left(\widetilde{X}_{N}^{\mathrm{f}}\right)-f\left(\widetilde{X}_{N}^{\mathrm{c}}\right)\right) .
$$

This description is for the case in which the timestep $h_{\ell}$ is halved on each successive level. There is a natural extension to other geometric sequences such as $h_{\ell}=4^{-\ell} h_{0}$, with the values of the coarse path drift and volatility being updated at the end of each coarse timestep, while the fine path values are updated after each fine path timestep.

\section{$4 \quad$ Numerical analysis}

We begin by making two sets of assumptions which will be assumed to hold throughout the analysis.

The first concerns the drift and volatility functions, and assumes a greater degree of smoothness than the usual assumptions used to prove half-order strong convergence for the Euler-Maruyama discretisation [27]. 
Assumption 4.1. The drift function $a: \mathbb{R} \rightarrow \mathbb{R}$ and volatility function $b: \mathbb{R} \rightarrow \mathbb{R}$ are both $C^{1}(\mathbb{R})$, and both they and their derivatives are Lipschitz continuous so that there exist constants $L_{a}, L_{b}, L_{a}^{\prime}, L_{b}^{\prime}$ such that

$$
\begin{array}{rlrl}
|a(x)-a(y)| & \leq L_{a}|x-y|, & |b(x)-b(y)| & \leq L_{b}|x-y|, \\
\left|a^{\prime}(x)-a^{\prime}(y)\right| & \leq L_{a}^{\prime}|x-y|, \quad\left|b^{\prime}(x)-b^{\prime}(y)\right| \leq L_{b}^{\prime}|x-y| .
\end{array}
$$

The second concerns approximate Normal random variables $\widetilde{Z}$, and their relationship to corresponding exact Normal random variables $Z$.

Assumption 4.2. Random variable pairs $(Z, \widetilde{Z})$ can be generated such that $Z \sim N(0,1)$, $\mathbb{E}[\widetilde{Z}]=0$, and $\mathbb{E}\left[|\widetilde{Z}-Z|^{p}\right] \leq \mathbb{E}\left[|Z|^{p}\right]$ for all $p \geq 2$.

In most cases, the pairs will be generated as $\left(\Phi^{-1}(U), \widetilde{Q}(U)\right)$, for a common uniform random variable $U$, but we leave open the possibility that they may be generated in an alternative way; this is needed later for Lemma 4.7. Note also that $|\widetilde{Z}| \leq|Z|+|\widetilde{Z}-Z|$, so a consequence of this assumption is that $\mathbb{E}\left[|\widetilde{Z}|^{p}\right] \leq 2^{p} \mathbb{E}\left[|Z|^{p}\right]$; this is also used later.

The proofs of the main results are greatly simplified by first proving the following lemma using the discrete Burkholder-Davis-Gundy inequality [28].

Lemma 4.3. Suppose that for a time interval $[0, T]$ with $T=N h$ and $t_{n}=n h$, we have the discrete equations

$$
D_{n+1}=D_{n}+A_{n} D_{n} h+B_{n} D_{n} h^{1 / 2} \widetilde{Z}_{n}+\Theta_{n} h+\Psi_{n} h^{1 / 2},
$$

subject to initial data $D_{0}=0$, where $\left(Z_{n}, \widetilde{Z}_{n}\right), n \geq 0$ are i.i.d. random variable pairs satisfying Assumption 4.2, and using the standard filtration $\mathcal{F}_{t}, A_{n}, B_{n}, \Theta_{n}$ are $\mathcal{F}_{t_{n}}$-adapted, depending only on $X_{0}$ and $\left(Z_{m}, \widetilde{Z}_{m}\right), m<n$, whereas $\Psi_{n}$ is $\mathcal{F}_{t_{n+1}}$-adapted with $\mathbb{E}\left[\Psi_{n} \mid \mathcal{F}_{t_{n}}\right]=0$.

Furthermore, suppose that $\left|A_{n}\right| \leq L_{a},\left|B_{n}\right| \leq L_{b}$, where $L_{a}, L_{b}$ are as defined in Assumption 4.1, and for some $p \geq 2$ there are constants $c_{1}, c_{2}$, which do not depend on $h$, such that

$$
\max _{0 \leq n<N} \mathbb{E}\left[\left|\Psi_{n}\right|^{p}\right] \leq c_{1}, \quad \max _{0 \leq n<N} \mathbb{E}\left[\left|\Theta_{n}\right|^{p}\right] \leq c_{2} .
$$

Then, there is a constant $c_{3}$ depending only on $L_{a}, L_{b}, p, T$ such that

$$
\mathbb{E}\left[\max _{0 \leq n \leq N}\left|D_{n}\right|^{p}\right] \leq c_{3}\left(c_{1}+c_{2}\right)
$$

Note: the lemma includes as a special case the case in which $\widetilde{Z}_{n}=Z_{n}$, i.e. with Normal random variables rather than approximate Normal random variables.

Proof. Summing over the first $n$ timesteps gives

$$
D_{n}=\sum_{m=0}^{n-1}\left\{A_{m} D_{m} h+B_{m} D_{m} h^{1 / 2} \widetilde{Z}_{m}+\Theta_{m} h+\Psi_{m} h^{1 / 2}\right\},
$$


and therefore if we define $E_{n} \equiv \mathbb{E}\left[\max _{0<n^{\prime} \leq n}\left|D_{n^{\prime}}\right|^{p}\right]$ we obtain, through Jensen's inequality,

$$
\begin{aligned}
E_{n} \leq & 4^{p-1} \mathbb{E}\left[\max _{n^{\prime} \leq n}\left|\sum_{m=0}^{n^{\prime}-1} A_{m} D_{m} h\right|^{p}\right]+4^{p-1} \mathbb{E}\left[\max _{n^{\prime} \leq n}\left|\sum_{m=0}^{n^{\prime}-1} B_{m} D_{m} h^{1 / 2} \widetilde{Z}_{m}\right|^{p}\right] \\
+ & 4^{p-1} \mathbb{E}\left[\max _{n^{\prime} \leq n}\left|\sum_{m=0}^{n^{\prime}-1} \Theta_{m} h\right|^{p}\right]+4^{p-1} \mathbb{E}\left[\max _{n^{\prime} \leq n}\left|\sum_{m=0}^{n^{\prime}-1} \Psi_{m} h^{1 / 2}\right|^{p}\right] .
\end{aligned}
$$

For the first term, using Jensen's inequality again gives

$$
\mathbb{E}\left[\max _{n^{\prime} \leq n}\left|\sum_{m=0}^{n^{\prime}-1} A_{m} D_{m} h\right|^{p}\right] \leq h^{p} n^{p-1} \sum_{m=0}^{n-1} \mathbb{E}\left[\left|A_{m} D_{m}\right|^{p}\right] \leq h T^{p-1} L_{a}^{p} \sum_{m=0}^{n-1} E_{m} .
$$

For the second term the discrete time Burkholder-Davis-Gundy inequality [28], together with Jensen's inequality and Assumption 4.2 gives the bound

$$
\begin{aligned}
\mathbb{E}\left[\max _{n^{\prime} \leq n}\left|\sum_{m=0}^{n^{\prime}-1} B_{m} D_{m} h^{1 / 2} \widetilde{Z}_{m}\right|^{p}\right] & \leq C_{p} \mathbb{E}\left[\left|\sum_{m=0}^{n-1} B_{m}^{2} D_{m}^{2} h \widetilde{Z}_{m}^{2}\right|^{p / 2}\right] \\
& \leq C_{p} h^{p / 2} n^{p / 2-1} \sum_{m=0}^{n-1} \mathbb{E}\left[\left|B_{m} D_{m}\right|^{p}\left|\widetilde{Z}_{m}\right|^{p}\right] \\
& \leq C_{p} h T^{p / 2-1} L_{b}^{p} 2^{p} \mathbb{E}\left[|Z|^{p}\right] \sum_{m=0}^{n-1} E_{m},
\end{aligned}
$$

with the constant $C_{p}$ depending only on $p$. Similarly, the third term has the bound

$$
\mathbb{E}\left[\max _{n^{\prime} \leq n}\left|\sum_{m=0}^{n^{\prime}-1} \Theta_{m} h\right|^{p}\right] \leq T^{p} c_{2}
$$

and the fourth term has the bound

$$
\mathbb{E}\left[\max _{n^{\prime} \leq n}\left|\sum_{m=0}^{n^{\prime}-1} \Psi_{m} h^{1 / 2}\right|^{p}\right] \leq C_{p} T^{p / 2} c_{1}
$$

Combining these four bounds we obtain

$$
\begin{aligned}
E_{n} \leq & 4^{p-1}\left(T^{p-1} L_{a}^{p}+C_{p} T^{p / 2-1} L_{b}^{p} 2^{p} \mathbb{E}\left[|Z|^{p}\right]\right) h \sum_{m=0}^{n-1} E_{m} \\
& +4^{p-1}\left(T^{p} c_{2}+C_{p} T^{p / 2} c_{1}\right)
\end{aligned}
$$

and therefore by Grönwall's inequality we obtain

$$
E_{n} \leq 4^{p-1}\left(T^{p} c_{2}+C_{p} T^{p / 2} c_{1}\right) \exp \left(4^{p-1}\left(T^{p} L_{a}^{p}+C_{p} 2^{p} \mathbb{E}\left[|Z|^{p}\right] T^{p / 2} L_{b}^{p}\right)\right) .
$$

Setting $c_{3}=4^{p-1} \max \left(T^{p}, C_{p} T^{p / 2}\right) \exp \left(4^{p-1}\left(T^{p} L_{a}^{p}+C_{p} 2^{p} \mathbb{E}\left[|Z|^{p}\right] T^{p / 2} L_{b}^{p}\right)\right)$ completes the proof. 
We are now ready to prove the first result, which is a generalisation of Lemma 2 in [17].

Lemma 4.4. For a fixed time interval $T=N h$, for any $p \geq 2$ there exists a constant $c$ which depends on $X_{0}, a, b, T, p$ but not on $h$ or $\mathbb{E}\left[|\widetilde{Z}-Z|^{p}\right]$, such that for any $0<n \leq N$

$$
\mathbb{E}\left[\max _{0 \leq n \leq N}\left|\widetilde{X}_{n}-\widehat{X}_{n}\right|^{p}\right] \leq c \mathbb{E}\left[|\widetilde{Z}-Z|^{p}\right]
$$

Proof. Taking the difference between

$$
\widetilde{X}_{n+1}=\widetilde{X}_{n}+a\left(\widetilde{X}_{n}\right) h+b\left(\widetilde{X}_{n}\right) h^{1 / 2} \widetilde{Z}_{n}
$$

and

$$
\widehat{X}_{n+1}=\widehat{X}_{n}+a\left(\widehat{X}_{n}\right) h+b\left(\widehat{X}_{n}\right) h^{1 / 2} Z_{n},
$$

and defining $D_{n} \equiv \widetilde{X}_{n}-\widehat{X}_{n}$, we obtain

$$
D_{n+1}=D_{n}+a^{\prime}\left(\xi_{1, n}\right) D_{n} h+b^{\prime}\left(\xi_{2, n}\right) D_{n} h^{1 / 2} \widetilde{Z}_{n}+b\left(\widehat{X}_{n}\right) h^{1 / 2}\left(\widetilde{Z}_{n}-Z_{n}\right)
$$

for suitably defined $\xi_{1, n}, \xi_{2, n}$ arising from the Mean Value Theorem, Lemma A.1.

This is in the correct form to apply Lemma 4.3 since $\left|a^{\prime}\left(\xi_{1, n}\right)\right| \leq L_{a}, \quad\left|b^{\prime}\left(\xi_{2, n}\right)\right| \leq L_{b}$,

$$
\mathbb{E}\left[b\left(\widehat{X}_{n}\right)\left(\widetilde{Z}_{n}-Z_{n}\right) \mid \widehat{X}_{n}\right]=b\left(\widehat{X}_{n}\right) \mathbb{E}\left[\widetilde{Z}_{n}-Z_{n}\right]=0
$$

and

$$
\left.\mathbb{E}\left[\left|b\left(\widehat{X}_{n}\right)\left(\widetilde{Z}_{n}-Z_{n}\right)\right|^{p}\right]=\left.\mathbb{E}\left[\left|b\left(\widehat{X}_{n}\right)\right|^{p}\right] \mathbb{E}\left[\mid \widetilde{Z}_{n}-Z_{n}\right)\right|^{p}\right] .
$$

The result then follows from Lemma 4.3 after noting that the standard analysis of the Euler-Maruyama method (e.g. see [27]) proves that $\mathbb{E}\left[\left|\widehat{X}_{n}\right|^{p}\right], \mathbb{E}\left[\left|a\left(\widehat{X}_{n}\right)\right|^{p}\right]$ and $\mathbb{E}\left[\left|b\left(\widehat{X}_{n}\right)\right|^{p}\right]$ are all uniformly bounded on the time interval $[0, T]$.

Corollary 4.5. For a fixed time interval $T=N h$, for any $p \geq 2$ there exists a constant $c$ which depends on $X_{0}, a, b, T, p$ but not on $h$, such that for any $0<n \leq N$

$$
\mathbb{E}\left[\left|\tilde{X}_{n}\right|^{p}\right] \leq c, \quad \mathbb{E}\left[\left|a\left(\widetilde{X}_{n}\right)\right|^{p}\right] \leq c, \quad \mathbb{E}\left[\left|b\left(\widetilde{X}_{n}\right)\right|^{p}\right] \leq c
$$

Proof. The standard analysis of the Euler-Maruyama method proves that $\mathbb{E}\left[\left|\widehat{X}_{n}\right|^{p}\right]$ is uniformly bounded on $[0, T]$, and so it follows from Lemma 4.4 that there exist constants $c_{1}$, $c_{2}$ such that

$$
\mathbb{E}\left[\left|\widetilde{X}_{n}\right|^{p}\right] \leq c_{1}+c_{2} \mathbb{E}\left[|\widetilde{Z}-Z|^{p}\right] \leq c_{1}+c_{2} \mathbb{E}\left[|Z|^{p}\right]
$$

due to Assumption 4.2. Since $\left|a\left(\widetilde{X}_{n}\right)\right| \leq|a(0)|+L_{a}\left|\widetilde{X}_{n}\right|$, it follows that

$$
\left|a\left(\widetilde{X}_{n}\right)\right|^{p} \leq 2^{p-1}\left(|a(0)|^{p}+L_{a}^{p}\left|\widetilde{X}_{n}\right|^{p}\right)
$$

and therefore $\mathbb{E}\left[\left|a\left(\widetilde{X}_{n}\right)\right|^{p}\right]$ can be uniformly bounded, and similarly $\mathbb{E}\left[\left|b\left(\widetilde{X}_{n}\right)\right|^{p}\right]$.

Corollary 4.6. For a fixed time interval $T=N h$, for any $p \geq 2$ there exists a constant $c$ which depends on $X_{0}, a, b, T, p$ but not on $h$ or $\mathbb{E}\left[|\widetilde{Z}-Z|^{p}\right]$ such that

$$
\max _{0 \leq n<N} \mathbb{E}\left[\left|\widetilde{X}_{n+1}-\widetilde{X}_{n}\right|^{p}\right] \leq c h^{p / 2}, \quad \max _{0 \leq n<N} \mathbb{E}\left[\left|\widehat{X}_{n+1}-\widehat{X}_{n}\right|^{p}\right] \leq c h^{p / 2}
$$

and

$$
\max _{0 \leq n<N} \mathbb{E}\left[\left|\left(\widetilde{X}_{n+1}-\widetilde{X}_{n}\right)-\left(\widehat{X}_{n+1}-\widehat{X}_{n}\right)\right|^{p}\right] \leq c h^{p / 2} \mathbb{E}\left[|\widetilde{Z}-Z|^{p}\right]
$$


Proof. Since

$$
\mathbb{E}\left[\left|\widetilde{X}_{n+1}-\widetilde{X}_{n}\right|^{p}\right] \leq 2^{p-1}\left(\mathbb{E}\left[\left|a\left(\widetilde{X}_{n}\right)\right|^{p}\right] h^{p}+\mathbb{E}\left[\left|b\left(\widetilde{X}_{n}\right)\right|^{p}\right] h^{p / 2} \mathbb{E}\left[\left|\widetilde{Z}_{n}\right|^{p}\right]\right)
$$

the first assertion follows from the uniform boundedness of $\mathbb{E}\left[\left|a\left(\widetilde{X}_{n}\right)\right|^{p}\right]$ and $\mathbb{E}\left[\left|b\left(\widetilde{X}_{n}\right)\right|^{p}\right]$, together with the trivial inequality $h^{p} \leq h^{p / 2} T^{p / 2}$ and the $\mathbb{E}\left[\left|\widetilde{Z}_{n}\right|^{p}\right]$ bound due to Assumption 4.2. The second assertion follows similarly.

Re-arranging Equation (7) gives

$$
D_{n+1}-D_{n}=a^{\prime}\left(\xi_{1, n}\right) D_{n} h+b^{\prime}\left(\xi_{2, n}\right) D_{n} h^{1 / 2} \widetilde{Z}_{n}+b\left(\widehat{X}_{n}\right) h^{1 / 2}\left(\widetilde{Z}_{n}-Z_{n}\right),
$$

with $D_{n} \equiv \widetilde{X}_{n}-\widehat{X}_{n}$. Hence,

$$
\begin{aligned}
& \mathbb{E}\left[\left|\left(\widetilde{X}_{n+1}-\widetilde{X}_{n}\right)-\left(\widehat{X}_{n+1}-\widehat{X}_{n}\right)\right|^{p}\right] \\
& \quad \leq 3^{p-1}\left(L_{a}^{p} \mathbb{E}\left[\left|D_{n}\right|^{p}\right] h^{p}+L_{b}^{p} \mathbb{E}\left[\left|D_{n}\right|^{p}\right] h^{p / 2} \mathbb{E}\left[\left|\widetilde{Z}_{n}\right|^{p}\right]+\mathbb{E}\left[\left|b\left(\widehat{X}_{n}\right)\right|^{p}\right] h^{p / 2} \mathbb{E}\left[|\widetilde{Z}-Z|^{p}\right]\right),
\end{aligned}
$$

and the third assertion follows from the bounds on $\mathbb{E}\left[\left|\widetilde{X}_{n}-\widehat{X}_{n}\right|^{p}\right]$, and $\mathbb{E}\left[\left|b\left(\widehat{X}_{n}\right)\right|^{p}\right]$.

We now have the first MLMC results involving level $\ell$ fine paths and level $\ell-1$ coarse paths, as defined in Equations (1)-(4).

Lemma 4.7. For a fixed time interval $T=N h$, for any $p \geq 2$ there exists a constant $c$ which depends on $X_{0}, a, b, T, p$, but not on $h$ or $\mathbb{E}\left[|\widetilde{Z}-Z|^{p}\right]$, such that for any $0<n \leq N$

$$
\mathbb{E}\left[\max _{0<n \leq N}\left|\widetilde{X}_{n}^{\mathrm{f}}-\widehat{X}_{n}^{\mathrm{f}}\right|^{p}\right] \leq c \mathbb{E}\left[|\widetilde{Z}-Z|^{p}\right], \quad \mathbb{E}\left[\max _{0<n \leq N}\left|\widetilde{X}_{n}^{\mathrm{c}}-\widehat{X}_{n}^{\mathrm{c}}\right|^{p}\right] \leq c \mathbb{E}\left[|\widetilde{Z}-Z|^{p}\right]
$$

Proof. The first assertion comes immediately from Lemma 4.4, but the second assertion requires the observation that if we set

$$
Z_{3}=\left(Z_{1}+Z_{2}\right) / \sqrt{2}, \quad \widetilde{Z}_{3}=\left(\widetilde{Z}_{1}+\widetilde{Z}_{2}\right) / \sqrt{2},
$$

where the independent pairs $\left(Z_{1}, \widetilde{Z}_{1}\right)$ and $\left(Z_{2}, \widetilde{Z}_{2}\right)$ satisfy Assumption 4.2 , then $Z_{3} \sim$ $N(0,1), \mathbb{E}\left[\widetilde{Z}_{3}\right]=0$, and by Jensen's inequality

$$
\mathbb{E}\left[\left|\widetilde{Z}_{3}-Z_{3}\right|^{p}\right] \leq 2^{p / 2-1}\left(\mathbb{E}\left[\left|\widetilde{Z}_{1}-Z_{1}\right|^{p}\right]+\mathbb{E}\left[\left|\widetilde{Z}_{2}-Z_{2}\right|^{p}\right]\right) \leq 2^{p / 2} \mathbb{E}\left[|Z|^{p}\right]
$$

Therefore the pair $\left(Z_{3}, \widetilde{Z}_{3}\right)$ also satisfies Assumption 4.2 apart from an increased bound on $\mathbb{E}\left[\left|\widetilde{Z}_{3}-Z_{3}\right|^{p}\right]$. This requires minor changes to the constants in the subsequent lemmas, but in the end the desired result follows from Lemma 4.4 .

Lemma 4.8. For a fixed time interval $T=N h$, for any $p \geq 2$ there exists a constant $c$ which depends on $X_{0}, a, b, T, p$, but not on $h$ or $\mathbb{E}\left[|\widetilde{Z}-Z|^{p}\right]$, such that for any $0<n \leq N$

$$
\mathbb{E}\left[\max _{0<n \leq N}\left|\widetilde{X}_{n}^{\mathrm{f}}-\widetilde{X}_{n}^{\mathrm{c}}\right|^{p}\right] \leq c h^{p / 2}, \quad \mathbb{E}\left[\max _{0<n \leq N}\left|\widehat{X}_{n}^{\mathrm{f}}-\widehat{X}_{n}^{\mathrm{c}}\right|^{p}\right] \leq c h^{p / 2},
$$


Proof. Defining $D_{n} \equiv \widetilde{X}_{n}^{\mathrm{f}}-\widetilde{X}_{n}^{\mathrm{c}}$, and taking the difference between equations (3) and (4) gives

$$
\begin{aligned}
D_{n+1}=D_{n} & +\left(a\left(\widetilde{X}_{n}^{\mathrm{f}}\right)-a\left(\widetilde{X}_{n}^{\mathrm{c}}\right)\right) h+\left(b\left(\widetilde{X}_{n}^{\mathrm{f}}\right)-b\left(\widetilde{X}_{n}^{\mathrm{c}}\right)\right) h^{1 / 2} \widetilde{Z}_{n} \\
& +\left(a\left(\widetilde{X}_{n}^{\mathrm{c}}\right)-a\left(\widetilde{X}_{\underline{n}}^{\mathrm{c}}\right)\right) h+\left(b\left(\widetilde{X}_{n}^{\mathrm{c}}\right)-b\left(\widetilde{X}_{\underline{n}}^{\mathrm{c}}\right)\right) h^{1 / 2} \widetilde{Z}_{n} \\
=D_{n} & +a^{\prime}\left(\xi_{1, n}\right) D_{n} h+b^{\prime}\left(\xi_{2, n}\right) D_{n} h^{1 / 2} \widetilde{Z}_{n} \\
& +a^{\prime}\left(\xi_{3, n}\right)\left(\widetilde{X}_{n}^{\mathrm{c}}-\widetilde{X}_{\underline{n}}^{\mathrm{c}}\right) h+b^{\prime}\left(\xi_{4, n}\right)\left(\widetilde{X}_{n}^{\mathrm{c}}-\widetilde{X}_{\underline{n}}^{\mathrm{c}}\right) h^{1 / 2} \widetilde{Z}_{n}
\end{aligned}
$$

for suitably defined $\xi_{1, n}, \xi_{2, n}, \xi_{3, n}, \xi_{4, n}$ arising from the Mean Value Theorem. Noting that

$$
\mathbb{E}\left[\left|a^{\prime}\left(\xi_{3, n}\right)\left(\widetilde{X}_{n}^{\mathrm{c}}-\widetilde{X}_{\underline{n}}^{\mathrm{c}}\right)\right|^{p}\right] \leq L_{a}^{p} \mathbb{E}\left[\left|\widetilde{X}_{n}^{\mathrm{c}}-\widetilde{X}_{\underline{n}}^{\mathrm{c}}\right|^{p}\right]
$$

and

$$
\mathbb{E}\left[\left|b^{\prime}\left(\xi_{4, n}\right)\left(\widetilde{X}_{n}^{\mathrm{c}}-\widetilde{X}_{\underline{n}}^{\mathrm{c}}\right) \widetilde{Z}_{n}\right|^{p}\right] \leq L_{b}^{p} \mathbb{E}\left[\left|\widetilde{X}_{n}^{\mathrm{c}}-\widetilde{X}_{\underline{n}}^{\mathrm{c}}\right|^{p}\right] \mathbb{E}\left[|\widetilde{Z}|^{p}\right] \leq L_{b}^{p} 2^{p} \mathbb{E}\left[|Z|^{p}\right] \mathbb{E}\left[\left|\widetilde{X}_{n}^{\mathrm{c}}-\widetilde{X}_{\underline{n}}^{\mathrm{c}}\right|^{p}\right],
$$

the first assertion then follows again from Lemma 4.3 after using the bounds for $\mathbb{E}\left[\left|\widetilde{X}_{n}^{\mathrm{c}}-\widetilde{X}_{\underline{n}}^{\mathrm{c}}\right|^{p}\right]$ which come from Corollary 4.6.

The second assertion follows similarly.

We now come to the analysis of the cross-difference.

Lemma 4.9. For a fixed time interval $T=N h$, for any $p, q$ with $2 \leq p<q$ there exists a constant $c$ which depends on $X_{0}, a, b, T$ but not on $h$ or $\mathbb{E}\left[|\widetilde{Z}-Z|^{q}\right]$, such that

$$
\mathbb{E}\left[\max _{0 \leq n<N}\left|\widetilde{X}_{n}^{\mathrm{f}}-\widetilde{X}_{n}^{\mathrm{c}}-\widehat{X}_{n}^{\mathrm{f}}+\widehat{X}_{n}^{\mathrm{c}}\right|^{p}\right] \leq c h^{p / 2}\left(\mathbb{E}\left[|\widetilde{Z}-Z|^{q}\right]\right)^{p / q} .
$$

Proof. Defining $D_{n} \equiv \widetilde{X}_{n}^{\mathrm{f}}-\widetilde{X}_{n}^{\mathrm{c}}-\widehat{X}_{n}^{\mathrm{f}}+\widehat{X}_{n}^{\mathrm{c}}$, then the difference of Equations (1)-(4) together with Lemma A.2 gives

$$
\begin{aligned}
& D_{n+1} \\
& =D_{n}+\left(a\left(\widetilde{X}_{n}^{\mathrm{f}}\right)-a\left(\widetilde{X}_{n}^{\mathrm{c}}\right)-a\left(\widehat{X}_{n}^{\mathrm{f}}\right)+a\left(\widehat{X}_{n}^{\mathrm{c}}\right)\right) h+\left(b\left(\widetilde{X}_{n}^{\mathrm{f}}\right)-b\left(\widetilde{X}_{n}^{\mathrm{c}}\right)-b\left(\widehat{X}_{n}^{\mathrm{f}}\right)+b\left(\widehat{X}_{n}^{\mathrm{c}}\right)\right) h^{1 / 2} \widetilde{Z}_{n} \\
& +\left(a\left(\widetilde{X}_{n}^{\mathrm{c}}\right)-a\left(\widetilde{X}_{\underline{n}}^{\mathrm{c}}\right)-a\left(\widehat{X}_{n}^{\mathrm{c}}\right)+a\left(\widehat{X}_{\underline{n}}^{\mathrm{c}}\right)\right) h+\left(b\left(\widetilde{X}_{n}^{\mathrm{c}}\right)-b\left(\widetilde{X}_{\underline{n}}^{\mathrm{c}}\right)-b\left(\widehat{X}_{n}^{\mathrm{c}}\right)+b\left(\widehat{X}_{\underline{n}}^{\mathrm{c}}\right)\right) h^{1 / 2} \widetilde{Z}_{n} \\
& +\left(b\left(\widehat{X}_{n}^{\mathrm{f}}\right)-b\left(\widehat{X}_{\underline{n}}^{\mathrm{c}}\right)\right) h^{1 / 2}\left(\widetilde{Z}_{n}-Z_{n}\right) \\
& =D_{n}+a^{\prime}\left(\xi_{1, n}\right) D_{n} h+b^{\prime}\left(\xi_{2, n}\right) D_{n} h^{1 / 2} \widetilde{Z}_{n} \\
& +a^{\prime}\left(\xi_{3, n}\right)\left(\widetilde{X}_{n}^{\mathrm{c}}-\widetilde{X}_{\underline{n}}^{\mathrm{c}}-\widehat{X}_{n}^{\mathrm{c}}+\widehat{X}_{\underline{n}}^{\mathrm{c}}\right) h+b^{\prime}\left(\xi_{4, n}\right)\left(\widetilde{X}_{n}^{\mathrm{c}}-\widetilde{X}_{\underline{n}}^{\mathrm{c}}-\widehat{X}_{n}^{\mathrm{c}}+\widehat{X}_{\underline{n}}^{\mathrm{c}}\right) h^{1 / 2} \widetilde{Z}_{n} \\
& +\left(R_{1, n}+R_{3, n}\right) h+\left(R_{2, n}+R_{4, n}\right) h^{1 / 2} \widetilde{Z}_{n}+b^{\prime}\left(\xi_{5, n}\right)\left(\widehat{X}_{n}^{\mathrm{f}}-\widehat{X}_{\underline{n}}^{\mathrm{c}}\right) h^{1 / 2}\left(\widetilde{Z}_{n}-Z_{n}\right)
\end{aligned}
$$

for suitably defined $\xi_{1, n}, \xi_{2, n}, \xi_{3, n}, \xi_{4, n}, \xi_{5, n}$ arising from Lemma A.2 and the Mean Value Theorem, and with

$$
\begin{aligned}
&\left|R_{1, n}\right| \leq \frac{1}{2} L_{a}^{\prime}\left(\left|\widetilde{X}_{n}^{\mathrm{f}}-\widetilde{X}_{n}^{\mathrm{c}}\right|+\left|\widehat{X}_{n}^{\mathrm{f}}-\widehat{X}_{n}^{\mathrm{c}}\right|\right)\left(\left|\widetilde{X}_{n}^{\mathrm{f}}-\widehat{X}_{n}^{\mathrm{f}}\right|+\left|\widetilde{X}_{n}^{\mathrm{c}}-\widehat{X}_{n}^{\mathrm{c}}\right|\right) \\
&\left|R_{2, n}\right| \leq \frac{1}{2} L_{b}^{\prime}\left(\left|\widetilde{X}_{n}^{\mathrm{f}}-\widetilde{X}_{n}^{\mathrm{c}}\right|+\left|\widehat{X}_{n}^{\mathrm{f}}-\widehat{X}_{n}^{\mathrm{c}}\right|\right)\left(\left|\widetilde{X}_{n}^{\mathrm{f}}-\widehat{X}_{n}^{\mathrm{f}}\right|+\left|\widetilde{X}_{n}^{\mathrm{c}}-\widehat{X}_{n}^{\mathrm{c}}\right|\right) \\
&\left|R_{3, n}\right| \leq \frac{1}{2} L_{a}^{\prime}\left(\left|\widetilde{X}_{n}^{\mathrm{c}}-\widetilde{X}_{\underline{n}}^{\mathrm{c}}\right|+\left|\widehat{X}_{n}^{\mathrm{c}}-\widehat{X}_{\underline{n}}^{\mathrm{c}}\right|\right)\left(\left|\widetilde{X}_{n}^{\mathrm{c}}-\widehat{X}_{n}^{\mathrm{c}}\right|+\left|\widetilde{X}_{\underline{n}}^{\mathrm{c}}-\widehat{X}_{\underline{n}}^{\mathrm{c}}\right|\right) \\
&\left|R_{4, n}\right| \leq \frac{1}{2} L_{b}^{\prime}\left(\left|\widetilde{X}_{n}^{\mathrm{c}}-\widetilde{X}_{\underline{n}}^{\mathrm{c}}\right|+\left|\widehat{X}_{n}^{\mathrm{c}}-\widehat{X}_{\underline{n}}^{\mathrm{c}}\right|\right)\left(\left|\widetilde{X}_{n}^{\mathrm{c}}-\widehat{X}_{n}^{\mathrm{c}}\right|+\left|\widetilde{X}_{\underline{n}}^{\mathrm{c}}-\widehat{X}_{\underline{n}}^{\mathrm{c}}\right|\right) .
\end{aligned}
$$


This equation is in the correct form for the application of Lemma 4.3 with

$$
\begin{aligned}
\Theta_{n}= & a^{\prime}\left(\xi_{3, n}\right)\left(\widetilde{X}_{n}^{\mathrm{c}}-\widetilde{X}_{\underline{n}}^{\mathrm{c}}-\widehat{X}_{n}^{\mathrm{c}}+\widehat{X}_{\underline{n}}^{\mathrm{c}}\right)+\left(R_{1, n}+R_{3, n}\right), \\
\Psi_{n}= & b^{\prime}\left(\xi_{4, n}\right)\left(\widetilde{X}_{n}^{\mathrm{c}}-\widetilde{X}_{\underline{n}}^{\mathrm{c}}-\widehat{X}_{n}^{\mathrm{c}}+\widehat{X}_{\underline{n}}^{\mathrm{c}}\right) \widetilde{Z}_{n}+\left(R_{2, n}+R_{4, n}\right) \widetilde{Z}_{n} \\
& +b^{\prime}\left(\xi_{5, n}\right)\left(\widehat{X}_{n}^{\mathrm{f}}-\widehat{X}_{\underline{n}}^{\mathrm{c}}\right)\left(\widetilde{Z}_{n}-Z_{n}\right) .
\end{aligned}
$$

Corollary 4.6 and Lemma 4.4 together with the Hölder inequality imply that there exists a constant $c$ such that

$$
\begin{aligned}
\mathbb{E}\left[\left|\widetilde{X}_{n}^{\mathrm{f}}-\widetilde{X}_{n}^{\mathrm{c}}\right|^{p}\left|\widetilde{X}_{n}^{\mathrm{f}}-\widehat{X}_{n}^{\mathrm{f}}\right|^{p}\right] & \leq\left(\mathbb{E}\left[\left|\widetilde{X}_{n}^{\mathrm{f}}-\widetilde{X}_{n}^{\mathrm{c}}\right|^{p /(1-p / q)}\right]\right)^{1-p / q}\left(\mathbb{E}\left[\left|\widetilde{X}_{n}^{\mathrm{f}}-\widehat{X}_{n}^{\mathrm{f}}\right|^{q}\right]\right)^{p / q} \\
& \leq c h^{p / 2}\left(\mathbb{E}\left[|\widetilde{Z}-Z|^{q}\right]\right)^{p / q}
\end{aligned}
$$

Bounding the other terms similarly, there is a different constant $c$ such that

$$
\mathbb{E}\left[\left|R_{i, n}\right|^{p}\right] \leq c h^{p / 2}\left(\mathbb{E}\left[|\widetilde{Z}-Z|^{q}\right]\right)^{p / q}, \quad i=1,2,3,4
$$

Due to Corollary 4.6 we also have

$$
\mathbb{E}\left[\left|\widetilde{X}_{n}^{\mathrm{c}}-\widetilde{X}_{\underline{n}}^{\mathrm{c}}-\widehat{X}_{n}^{\mathrm{c}}+\widehat{X}_{\underline{n}}^{\mathrm{c}}\right|^{p}\right] \leq c h^{p / 2}\left(\mathbb{E}\left[|\widetilde{Z}-Z|^{q}\right]\right)^{p / q}
$$

for some constant $c$, and finally, for another constant $c$,

$$
\mathbb{E}\left[\left|\left(\widehat{X}_{n}^{\mathrm{f}}-\widehat{X}_{\underline{n}}^{\mathrm{c}}\right)\left(\widetilde{Z}_{n}-Z_{n}\right)\right|^{p}\right]=\mathbb{E}\left[\left|\widehat{X}_{n}^{\mathrm{f}}-\widehat{X}_{\underline{n}}^{\mathrm{c}}\right|^{p}\right] \mathbb{E}\left[|\widetilde{Z}-Z|^{p}\right] \leq c h^{p / 2}\left(\mathbb{E}\left[|\widetilde{Z}-Z|^{q}\right]\right)^{p / q} .
$$

Hence, we end up concluding that there exists another constant $c$ such that

$$
\mathbb{E}\left[\left|\Psi_{n}\right|^{p}\right] \leq c h^{p / 2}\left(\mathbb{E}\left[|\widetilde{Z}-Z|^{q}\right]\right)^{p / q}, \quad \mathbb{E}\left[\left|\Theta_{n}\right|^{p}\right] \leq c h^{p / 2}\left(\mathbb{E}\left[|\widetilde{Z}-Z|^{q}\right]\right)^{p / q}
$$

and then Lemma 4.3 gives us the desired final result.

We now obtain a lemma for output functions $f(x)$ which are locally Lipschitz with at worst a polynomial growth as $|x| \rightarrow \infty$.

Lemma 4.10. For a fixed time interval $T=N$, if the function $f: \mathbb{R} \rightarrow \mathbb{R}$ is $C^{1}(\mathbb{R})$ and there is an exponent $r>0$ and constants $L_{f}, L_{f}^{\prime}$ such that

$$
|f(x)-f(y)| \leq L_{f}\left(1+|x|^{r}+|y|^{r}\right)|x-y|, \quad\left|f^{\prime}(x)-f^{\prime}(y)\right| \leq L_{f}^{\prime}\left(1+|x|^{r}+|y|^{r}\right)|x-y|,
$$

then for any $q>2$ there exists a constant $c$ which depends on $X_{0}, a, b, f, T$, but not on $h$ or $\mathbb{E}\left[|\widetilde{Z}-Z|^{q}\right]$, such that

$$
\mathbb{V}\left[f\left(\widehat{X}_{N}^{\mathrm{f}}\right)-f\left(\widehat{X}_{N}^{\mathrm{c}}\right)-f\left(\widetilde{X}_{N}^{\mathrm{f}}\right)+f\left(\widetilde{X}_{N} c\right)\right] \leq c h\left(\mathbb{E}\left[|\widetilde{Z}-Z|^{q}\right]\right)^{2 / q} .
$$

Proof. Given the assumptions on $f$, we are able to follow the proof of Lemma A.2 to obtain

$$
f\left(\widehat{X}_{N}^{\mathrm{f}}\right)-f\left(\widehat{X}_{N}^{\mathrm{c}}\right)-f\left(\widetilde{X}_{N}^{\mathrm{f}}\right)+f\left(\widetilde{X}_{N}^{\mathrm{c}}\right)=f^{\prime}(\xi)\left(\widehat{X}_{N}^{\mathrm{f}}-\widehat{X}_{N}^{\mathrm{c}}-\widetilde{X}_{N}^{\mathrm{f}}+\widetilde{X}_{N}^{\mathrm{c}}\right)+R,
$$


where

$$
\left|f^{\prime}(\xi)\right| \leq L_{f}\left(1+\left|\widehat{X}_{N}^{\mathrm{f}}\right|^{r}+\left|\widehat{X}_{N}^{\mathrm{c}}\right|^{r}+\left|\widetilde{X}_{N}^{\mathrm{f}}\right|^{r}+\left|\widetilde{X}_{N}^{\mathrm{c}}\right|^{r}\right)
$$

and

$$
\begin{aligned}
|R| \leq & \frac{1}{2} L_{f}^{\prime}\left(1+\left|\widehat{X}_{N}^{\mathrm{f}}\right|^{r}+\left|\widehat{X}_{N}^{\mathrm{c}}\right|^{r}+\left|\widetilde{X}_{N}^{\mathrm{f}}\right|^{r}+\left|\widetilde{X}_{N}^{\mathrm{c}}\right|^{r}\right) \\
& \times\left(\left|\widehat{X}_{N}^{\mathrm{f}}-\widehat{X}_{N}^{\mathrm{c}}\right|+\left|\widetilde{X}_{N}^{\mathrm{f}}-\widetilde{X}_{N}^{\mathrm{c}}\right|\right)\left(\left|\widehat{X}_{N}^{\mathrm{f}}-\widetilde{X}_{N}^{\mathrm{f}}\right|+\left|\widehat{X}_{N}^{\mathrm{c}}-\widetilde{X}_{N}^{\mathrm{c}}\right|\right) .
\end{aligned}
$$

Hence,

$$
\mathbb{V}\left[f\left(\widehat{X}_{N}^{\mathrm{f}}\right)-f\left(\widehat{X}_{N}^{\mathrm{c}}\right)-f\left(\widetilde{X}_{N}^{\mathrm{f}}\right)+f\left(\widetilde{X}_{N}^{\mathrm{c}}\right)\right] \leq 2 \mathbb{E}\left[\left(f^{\prime}(\xi)\right)^{2}\left|\widehat{X}_{N}^{\mathrm{f}}-\widehat{X}_{N}^{\mathrm{c}}-\widetilde{X}_{N}^{\mathrm{f}}+\widetilde{X}_{N}^{\mathrm{c}}\right|^{2}\right]+2 \mathbb{E}\left[R^{2}\right] .
$$

Due to Hölder's inequality,

$$
\begin{aligned}
& \mathbb{E}\left[\left|\widehat{X}_{N}^{\mathrm{f}}\right|^{2 r}\left|\widehat{X}_{N}^{\mathrm{f}}-\widehat{X}_{N}^{\mathrm{c}}\right|^{2}\left|\widehat{X}_{N}^{\mathrm{f}}-\widetilde{X}_{N}^{\mathrm{f}}\right|^{2}\right] \\
& \quad \leq\left(\mathbb{E}\left[\left|\widehat{X}_{N}^{\mathrm{f}}\right|^{2 r /(1 / 2-1 / q)}\right]\right)^{1 / 2-1 / q}\left(\mathbb{E}\left[\left|\widehat{X}_{N}^{\mathrm{f}}-\widehat{X}_{N}^{\mathrm{c}}\right|^{2 /(1 / 2-1 / q)}\right]\right)^{1 / 2-1 / q}\left(\mathbb{E}\left[\left|\widehat{X}_{N}^{\mathrm{f}}-\widetilde{X}_{N}^{\mathrm{f}}\right|^{q}\right]\right)^{2 / q}
\end{aligned}
$$

Note that $\mathbb{E}\left[\left|\widehat{X}_{N}^{\mathrm{f}}\right|^{2 r /(1 / 2-1 / q)}\right]$ is finite and uniformly bounded due to Corollary 4.5. The other terms in $\mathbb{E}\left[R^{2}\right]$ can be bounded similarly, and therefore due to the bounds from Lemmas 4.4 and 4.8 there exists a constant $c$, not depending on $h$ or $\mathbb{E}\left[|\widetilde{Z}-Z|^{q}\right]$, such that

$$
\mathbb{E}\left[R^{2}\right] \leq \operatorname{ch}\left(\mathbb{E}\left[|\widetilde{Z}-Z|^{q}\right]\right)^{2 / q} .
$$

Similarly, choosing $p$ such that $2<p<q$, then due to Hölder's inequality,

$$
\begin{aligned}
\mathbb{E} & {\left[\left(f^{\prime}(\xi)\right)^{2}\left|\widehat{X}_{N}^{\mathrm{f}}-\widehat{X}_{N}^{\mathrm{c}}-\widetilde{X}_{N}^{\mathrm{f}}+\widetilde{X}_{N}^{\mathrm{c}}\right|^{2}\right] } \\
& \leq\left(\mathbb{E}\left[\left|f^{\prime}(\xi)\right|^{2 /(1-2 / p)}\right]\right)^{1-2 / p}\left(\mathbb{E}\left[\left|\widehat{X}_{N}^{\mathrm{f}}-\widehat{X}_{N}^{\mathrm{c}}-\widetilde{X}_{N}^{\mathrm{f}}+\widetilde{X}_{N}^{\mathrm{c}}\right|^{p}\right]\right)^{2 / p}
\end{aligned}
$$

$\mathbb{E}\left[\left|f^{\prime}(\xi)\right|^{2 /(1-2 / p)}\right]$ is finite and uniformly bounded due to Corollary 4.5 , and therefore the bound for $\mathbb{E}\left[\left|\widehat{X}_{N}^{\mathrm{f}}-\widehat{X}_{N}^{\mathrm{c}}-\widetilde{X}_{N}^{\mathrm{f}}+\widetilde{X}_{N}^{\mathrm{c}}\right|^{p}\right]$ from Lemma 4.9 completes the proof.

In finance applications, put and call options correspond to $f(x) \equiv \max (K-x, 0)$ and $\max (x-K, 0)$, respectively, with $K>0$ being the "strike". More generally, we can consider functions $f$ which are globally Lipschitz with a derivative which exists and is continuous everywhere except at a single point $K$.

Heuristically, the four values $\widehat{X}_{N}^{\mathrm{f}}, \widehat{X}_{N}^{\mathrm{c}}, \widetilde{X}_{N}^{\mathrm{f}}, \widetilde{X}_{N}^{\mathrm{c}}$ do not differ from each other, or from $X_{T}$, by more than $O\left(\max \left\{h^{1 / 2},\left(\mathbb{E}\left[|\widetilde{Z}-Z|^{2}\right]\right)^{1 / 2}\right\}\right)$. If $X_{T}$ has a bounded probability density, then the probability that $X_{T}$ is within this distance of $K$ is $O\left(\max \left\{h^{1 / 2},\left(\mathbb{E}\left[|\widetilde{Z}-Z|^{2}\right]\right)^{1 / 2}\right\}\right)$, and in this case the global Lipschitz property for $f$ gives

$$
f\left(\widehat{X}_{N}^{\mathrm{f}}\right)-f\left(\widehat{X}_{N}^{\mathrm{c}}\right)-f\left(\widetilde{X}_{N}^{\mathrm{f}}\right)+f\left(\widetilde{X}_{N}^{\mathrm{c}}\right)=O\left(\min \left\{h^{1 / 2},\left(\mathbb{E}\left[|\widetilde{Z}-Z|^{2}\right]\right)^{1 / 2}\right\}\right),
$$

with the first term in the minimum coming from

$$
f\left(\widehat{X}_{N}^{\mathrm{f}}\right)-f\left(\widehat{X}_{N}^{\mathrm{c}}\right)-f\left(\widetilde{X}_{N}^{\mathrm{f}}\right)+f\left(\widetilde{X}_{N}^{\mathrm{c}}\right)=\left(f\left(\widehat{X}_{N}^{\mathrm{f}}\right)-f\left(\widehat{X}_{N}^{\mathrm{c}}\right)\right)-\left(f\left(\widetilde{X}_{N}^{\mathrm{f}}\right)+f\left(\widetilde{X}_{N}^{\mathrm{c}}\right)\right)
$$

together with Lemma 4.8, while the second term comes from

$$
f\left(\widehat{X}_{N}^{\mathrm{f}}\right)-f\left(\widehat{X}_{N}^{\mathrm{c}}\right)-f\left(\widetilde{X}_{N}^{\mathrm{f}}\right)+f\left(\widetilde{X}_{N}^{\mathrm{c}}\right)=\left(f\left(\widehat{X}_{N}^{\mathrm{f}}\right)-f\left(\widetilde{X}_{N}^{\mathrm{f}}\right)\right)-\left(f\left(\widehat{X}_{N}^{\mathrm{c}}\right)+f\left(\widetilde{X}_{N}^{\mathrm{c}}\right)\right)
$$


together with Lemma 4.4 .

On the other hand, if $X_{T}$ is more than this distance from $K$ then all four values will be on the same side of $K$ and then

$$
f\left(\widehat{X}_{N}^{\mathrm{f}}\right)-f\left(\widehat{X}_{N}^{\mathrm{c}}\right)-f\left(\widetilde{X}_{N}^{\mathrm{f}}\right)+f\left(\widetilde{X}_{N}^{\mathrm{c}}\right)=O\left(h^{1 / 2}\left(\mathbb{E}\left[|\widetilde{Z}-Z|^{2}\right]\right)^{1 / 2}\right) .
$$

Consequently,

$$
\begin{aligned}
\mathbb{V} & {\left[f\left(\widehat{X}_{N}^{\mathrm{f}}\right)-f\left(\widehat{X}_{N}^{\mathrm{c}}\right)-f\left(\widetilde{X}_{N}^{\mathrm{f}}\right)+f\left(\widetilde{X}_{N}^{\mathrm{c}}\right)\right] } \\
& =O\left(\max \left\{h^{1 / 2},\left(\mathbb{E}\left[|\widetilde{Z}-Z|^{2}\right]\right)^{1 / 2}\right\}\right) \times O\left(\min \left\{h, \mathbb{E}\left[|\widetilde{Z}-Z|^{2}\right]\right\}\right)+O\left(h \mathbb{E}\left[|\widetilde{Z}-Z|^{2}\right]\right) \\
& =O\left(\min \left\{h\left(\mathbb{E}\left[|\widetilde{Z}-Z|^{2}\right]\right)^{1 / 2}, h^{1 / 2} \mathbb{E}\left[|\widetilde{Z}-Z|^{2}\right]\right\}\right) .
\end{aligned}
$$

The bound in the following lemma (for the case $q \approx 2$ ) is slightly weaker, but the proof follows along similar lines in establishing that the dominant contribution to the variance comes from samples with $X_{T}$ near $K$.

In the proof, we will use the notation

$$
g_{1}\left(h, \mathbb{E}\left[|\widetilde{Z}-Z|^{q}\right]\right) \prec g_{2}\left(h, \mathbb{E}\left[|\widetilde{Z}-Z|^{q}\right]\right)
$$

for any two strictly positive functions $g_{1}, g_{2}$ to mean that there exists a constant $c>0$ which does not depend on $h$ or $\mathbb{E}\left[|\widetilde{Z}-Z|^{q}\right]$ such that

$$
g_{1}\left(h, \mathbb{E}\left[|\widetilde{Z}-Z|^{q}\right]\right)<c g_{2}\left(h, \mathbb{E}\left[|\widetilde{Z}-Z|^{q}\right]\right) .
$$

Note that if $0<a<b$ then

$$
h^{b} \leq T^{b-a} h^{a} \quad \Longrightarrow \quad h^{b} \prec h^{a}
$$

and likewise, due to Assumption 4.2,

$$
\left(\mathbb{E}\left[|\widetilde{Z}-Z|^{q}\right]\right)^{b} \leq\left(\mathbb{E}\left[|Z|^{q}\right]\right)^{b-a}\left(\mathbb{E}\left[|\widetilde{Z}-Z|^{q}\right]\right)^{a} \Longrightarrow\left(\mathbb{E}\left[|\widetilde{Z}-Z|^{q}\right]\right)^{b} \prec\left(\mathbb{E}\left[|\widetilde{Z}-Z|^{q}\right]\right)^{a} .
$$

Lemma 4.11. Suppose that the conditions of Lemma 4.10 are slightly modified so that $f: \mathbb{R} \rightarrow \mathbb{R}$ is $C^{1}(\mathbb{R} \backslash K)$ and there is an exponent $r>0$ and constants $L_{f}, L_{f}^{\prime}$ such

$$
\begin{aligned}
|f(x)-f(y)| & \leq L_{f}\left(1+|x|^{r}+|y|^{r}\right)|x-y|, & & \text { for all } x, y \\
\left|f^{\prime}(x)-f^{\prime}(y)\right| & \leq L_{f}^{\prime}\left(1+|x|^{r}+|y|^{r}\right)|x-y|, & & \text { if } \underline{\text { either }} x>y>K \text { or } x<y<K,
\end{aligned}
$$

and furthermore $X_{T}$ has a bounded probability density in the neighbourhood of $K$ and therefore there is a constant $c_{\rho}>0$ such that for any $D>0$

$$
\mathbb{P}\left[\left|X_{T}-K\right|<D\right] \leq c_{\rho} D
$$

Then for any $q>2$ and any $\delta>0$, there exists a constant $c_{\delta}$ which depends on $X_{0}, a, b$, $f, T, q$ and $\delta$, but not on $h$ or $\mathbb{E}\left[|\widetilde{Z}-Z|^{q}\right]$, such that

$$
\begin{aligned}
\mathbb{V}\left[f\left(\widehat{X}_{N}^{\mathrm{f}}\right)-f\left(\widehat{X}_{N}^{\mathrm{c}}\right)-f\left(\widetilde{X}_{N}^{\mathrm{f}}\right)+f\left(\widetilde{X}_{N}^{\mathrm{c}}\right)\right] \\
\leq c_{\delta} \min \left\{h\left(\mathbb{E}\left[|\widetilde{Z}-Z|^{q}\right]\right)^{(1-\delta) /(q+1)}, h^{(1-\delta) / 2-1 / q}\left(\mathbb{E}\left[|\widetilde{Z}-Z|^{q}\right]\right)^{2 / q}\right\}
\end{aligned}
$$


Proof. The proof follows an approach used previously in the analysis of MLMC variance for similar options in the context of multi-dimensional SDEs (Theorem 5.2 in [29]).

The proof is given for $0<\delta<1-1 / q$. If the assertion is true for $\delta$ in this range then it also holds for larger values.

If we define the events $A$ and $B$ as

$$
A:\left|X_{T}-K\right| \leq D, \quad B: \max \left\{\left|\widehat{X}_{N}^{\mathrm{f}}-X_{T}\right|,\left|\widehat{X}_{N}^{\mathrm{c}}-X_{T}\right|,\left|\widetilde{X}_{N}^{\mathrm{f}}-\widehat{X}_{N}^{\mathrm{f}}\right|,\left|\widetilde{X}_{N}^{\mathrm{c}}-\widehat{X}_{N}^{\mathrm{c}}\right|,\right\} \geq D / 2,
$$

for some choice of constant $D>0$, and define $\Delta f \equiv f\left(\widehat{X}_{N}^{\mathrm{f}}\right)-f\left(\widehat{X}_{N}^{\mathrm{c}}\right)-f\left(\widetilde{X}_{N}^{\mathrm{f}}\right)+f\left(\widetilde{X}_{N}^{\mathrm{c}}\right)$, then

$$
\mathbb{V}[\Delta f] \leq \mathbb{E}\left[(\Delta f)^{2} \mathbf{1}_{A \cup B}\right]+\mathbb{E}\left[(\Delta f)^{2} \mathbf{1}_{A^{c} \cap B^{c}}\right]
$$

where $A^{c}, B^{c}$ are the complements of $A$ and $B$, and $\mathbf{1}_{C}$ is the indicator function which has value 1 if the random sample $\omega \in C$, and 0 otherwise. Note that if $\omega \in A^{c} \cap B^{c}$ then the four values $\widehat{X}_{N}^{\mathrm{f}}, \widehat{X}_{N}^{\mathrm{c}}, \widetilde{X}_{N}^{\mathrm{f}}, \widetilde{X}_{N}^{\mathrm{c}}$ are all on the same side of $K$, and therefore the proof in Lemma 4.10 means that

$$
\mathbb{E}\left[(\Delta f)^{2} \mathbf{1}_{A^{c} \cap B^{c}}\right] \prec h\left(\mathbb{E}\left[|\widetilde{Z}-Z|^{q}\right]\right)^{2 / q} \prec h\left(\mathbb{E}\left[|\widetilde{Z}-Z|^{q}\right]\right)^{(1-\delta) /(q+1)}
$$

so $\mathbb{E}\left[(\Delta f)^{2} \mathbf{1}_{A^{c} \cap B^{c}}\right]$ is not the dominant contributor to the bound in (8).

To address the other term, $\mathbb{E}\left[(\Delta f)^{2} \mathbf{1}_{A \cup B}\right]$ we begin by noting that the two terms in the bound on the r.h.s. of $(8)$ are equal when $h^{1 / 2}=\left(\mathbb{E}\left[|\widetilde{Z}-Z|^{q}\right]\right)^{1 /(q+1)}$.

Case A: $h^{1 / 2} \leq\left(\mathbb{E}\left[|\widetilde{Z}-Z|^{q}\right]\right)^{1 /(q+1)}$.

In this case, we set $D=\left(\mathbb{E}\left[|\widetilde{Z}-Z|^{q}\right]\right)^{(1-\delta / 2) /(q+1)}$, and by Hölder's inequality we have

$$
\mathbb{E}\left[(\Delta f)^{2} \mathbf{1}_{A \cup B}\right] \leq\left(\mathbb{E}\left[|\Delta f|^{2 / \delta^{\prime}}\right]\right)^{\delta^{\prime}}\left(\mathbb{E}\left[\mathbf{1}_{A \cup B}\right]\right)^{1-\delta^{\prime}} \leq\left(\mathbb{E}\left[|\Delta f|^{2 / \delta^{\prime}}\right]\right)^{\delta^{\prime}}(\mathbb{P}[A]+\mathbb{P}[B])^{1-\delta^{\prime}},
$$

where $\delta^{\prime}=\delta /(2-\delta)$ so that $1-\delta^{\prime}=(1-\delta) /(1-\delta / 2)$.

Due to the assumed bounded density for $X_{T}$, we have $\mathbb{P}[A] \prec D$. Also,

$$
\begin{aligned}
\mathbb{P}[B] \leq & \mathbb{P}\left[\left|\widehat{X}_{N}^{\mathrm{f}}-X_{T}\right|>D / 2\right]+\mathbb{P}\left[\left|\widehat{X}_{N}^{\mathrm{c}}-X_{T}\right|>D / 2\right] \\
+ & \mathbb{P}\left[\left|\widetilde{X}_{N}^{\mathrm{f}}-\widehat{X}_{N}^{\mathrm{f}}\right|>D / 2\right]+\mathbb{P}\left[\left|\widetilde{X}_{N}^{\mathrm{c}}-\widehat{X}_{N}^{\mathrm{c}}\right|>D / 2\right]
\end{aligned}
$$

By the Markov inequality, together with the standard strong convergence results,

$$
\mathbb{P}\left[\left|\widehat{X}_{N}^{\mathrm{f}}-X_{T}\right|>D / 2\right] \leq \frac{\mathbb{E}\left[\left|\widehat{X}_{N}^{\mathrm{f}}-X_{T}\right|^{p}\right]}{(D / 2)^{p}} \prec \frac{h^{p / 2}}{D^{p}} \prec\left(\mathbb{E}\left[|\widetilde{Z}-Z|^{q}\right]\right)^{p \delta /(2 q+2)} \prec D,
$$

by choosing $p>2 / \delta-1$. A similar bound follows for $\mathbb{P}\left[\left|\widehat{X}_{N}^{\mathrm{c}}-X_{T}\right|>D / 2\right]$. In addition, the Markov inequality, together with Lemma 4.4, gives

$$
\mathbb{P}\left[\left|\widetilde{X}_{N}^{\mathrm{f}}-\widehat{X}_{N}^{\mathrm{f}}\right|>D / 2\right] \leq \frac{\mathbb{E}\left[\left|\widetilde{X}_{N}^{\mathrm{f}}-\widehat{X}_{N}^{\mathrm{f}}\right|^{q}\right]}{D^{q}} \prec \frac{\mathbb{E}\left[|\widetilde{Z}-Z|^{q}\right]}{D^{q}} \prec D
$$

and a similar bound holds for $\mathbb{P}\left[\left|\widetilde{X}_{N}^{\mathrm{c}}-\widehat{X}_{N}^{\mathrm{c}}\right|>D / 2\right]$. The conclusion from this is that $\mathbb{P}[B] \prec D$. 
Hence,

$$
(\mathbb{P}[A]+\mathbb{P}[B])^{(1-\delta) /(1-\delta / 2)} \prec D^{(1-\delta) /(1-\delta / 2)}=\left(\mathbb{E}\left[|\widetilde{Z}-Z|^{q}\right]\right)^{(1-\delta) /(q+1)} .
$$

In addition, we have

$$
|\Delta f|^{2 / \delta^{\prime}} \leq 2^{2 / \delta^{\prime}-1}\left(\left|f\left(\widehat{X}_{N}^{\mathrm{f}}\right)-f\left(\widehat{X}_{N}^{\mathrm{c}}\right)\right|^{2 / \delta^{\prime}}+\left|f\left(\widetilde{X}_{N}^{\mathrm{f}}\right)-f\left(\widetilde{X}_{N}^{\mathrm{c}}\right)\right|^{2 / \delta^{\prime}}\right)
$$

and due to Hölder's inequality and the bounds in Corollary 4.5 and Lemma 4.8 we have

$$
\begin{aligned}
\mathbb{E}\left[\left|f\left(\widetilde{X}_{N}^{\mathrm{f}}\right)-f\left(\widetilde{X}_{N}^{\mathrm{c}}\right)\right|^{2 / \delta^{\prime}}\right] & \leq L_{f}^{2 / \delta^{\prime}}\left(\mathbb{E}\left[\left.|1+c| \widetilde{X}_{N}^{\mathrm{f}}\right|^{r}+\left.c\left|\widetilde{X}_{N}^{\mathrm{c}}\right|^{r}\right|^{4 / \delta^{\prime}}\right]\right)^{1 / 2}\left(\mathbb{E}\left[\left|\widetilde{X}_{N}^{\mathrm{f}}-\widetilde{X}_{N}^{\mathrm{c}}\right|^{4 / \delta^{\prime}}\right]\right)^{1 / 2} \\
& \prec h^{1 / \delta^{\prime}} .
\end{aligned}
$$

There is a similar bound for $\mathbb{E}\left[\left|f\left(\widehat{X}_{N}^{\mathrm{f}}\right)-f\left(\widehat{X}_{N}^{\mathrm{c}}\right)\right|^{2 / \delta^{\prime}}\right]$ and hence we have the result that $\mathbb{E}\left[(\Delta f)^{2} \mathbf{1}_{A \cup B}\right] \prec h\left(\mathbb{E}\left[|\widetilde{Z}-Z|^{q}\right]\right)^{(1-\delta) /(q+1)}$ when $h^{1 / 2} \leq\left(\mathbb{E}\left[|\widetilde{Z}-Z|^{q}\right]\right)^{1 /(q+1)}$.

Case B: $h^{1 / 2} \geq\left(\mathbb{E}\left[|\widetilde{Z}-Z|^{q}\right]\right)^{1 /(q+1)}$.

In this case we set $D=h^{(1-\delta) / 2}$ and by Hölder's inequality we have

$$
\begin{aligned}
\mathbb{E}\left[(\Delta f)^{2} \mathbf{1}_{A \cup B}\right] & \leq\left(\mathbb{E}\left[|\Delta f|^{2 /\left(2 / q+\delta^{\prime}\right)}\right]\right)^{2 / q+\delta^{\prime}}\left(\mathbb{E}\left[\mathbf{1}_{A \cup B}\right]\right)^{1-2 / q-\delta^{\prime}} \\
& \leq\left(\mathbb{E}\left[|\Delta f|^{2 /\left(2 / q+\delta^{\prime}\right)}\right]\right)^{2 / q+\delta^{\prime}}(\mathbb{P}[A]+\mathbb{P}[B])^{1-2 / q-\delta^{\prime}},
\end{aligned}
$$

where $\delta^{\prime}=2 \delta /(q(1-\delta))$ so that $\left(1-2 / q-\delta^{\prime}\right)(1-\delta) / 2=(1-\delta) / 2-1 / q$.

We again have $\mathbb{P}[A] \prec D$. By the Markov inequality, together with the standard strong convergence results,

$$
\mathbb{P}\left[\left|\widehat{X}_{N}^{\mathrm{f}}-X_{T}\right|>D / 2\right] \leq \frac{\mathbb{E}\left[\left|\widehat{X}_{N}^{\mathrm{f}}-X_{T}\right|^{p}\right]}{(D / 2)^{p}} \prec \frac{h^{p / 2}}{h^{p(1-\delta) / 2}}=h^{p \delta / 2} \prec D,
$$

by choosing $p>1 / \delta$, and a similar bound follows for $\mathbb{P}\left[\left|\widehat{X}_{N}^{\mathrm{c}}-X_{T}\right|>D / 2\right]$. In addition, the Markov inequality, together with Lemma 4.4, gives

$$
\mathbb{P}\left[\left|\widetilde{X}_{N}^{\mathrm{f}}-\widehat{X}_{N}^{\mathrm{f}}\right|>D / 2\right] \leq \frac{\mathbb{E}\left[\left|\widetilde{X}_{N}^{\mathrm{f}}-\widehat{X}_{N}^{\mathrm{f}}\right|^{q}\right]}{D^{q}} \prec \frac{\mathbb{E}\left[|\widetilde{Z}-Z|^{q}\right]}{D^{q}} \prec \frac{h^{(q+1) / 2}}{h^{q(1-\delta) / 2}}=h^{1 / 2+q \delta / 2} \prec D,
$$

and a similar bound holds for $\mathbb{P}\left[\left|\widetilde{X}_{N}^{\mathrm{c}}-\widehat{X}_{N}^{\mathrm{c}}\right|>D / 2\right]$. The conclusion from this is that $\mathbb{P}[B] \prec D$, as before, and so

$$
(\mathbb{P}[A]+\mathbb{P}[B])^{1-2 / q-\delta^{\prime}} \prec D^{1-2 / q-\delta^{\prime}}=h^{\left(1-2 / q-\delta^{\prime}\right)(1-\delta) / 2}=h^{(1-\delta) / 2-1 / q} .
$$

In addition, defining $\delta^{\prime \prime}=\delta^{\prime} /\left(2 / q+\delta^{\prime}\right)$ so that $2 /\left(2 / q+\delta^{\prime}\right)=q\left(1-\delta^{\prime \prime}\right)$, we have

$$
|\Delta f|^{q\left(1-\delta^{\prime \prime}\right)} \leq 2^{q\left(1-\delta^{\prime \prime}\right)-1}\left(\left|f\left(\widetilde{X}_{N}^{\mathrm{f}}\right)-f\left(\widehat{X}_{N}^{\mathrm{f}}\right)\right|^{q\left(1-\delta^{\prime \prime}\right)}+\left|f\left(\widetilde{X}_{N}^{\mathrm{c}}\right)-f\left(\widehat{X}_{N}^{\mathrm{c}}\right)\right|^{q\left(1-\delta^{\prime \prime}\right)}\right)
$$

and due to Hölder's inequality and the bounds in Corollary 4.5 and Lemma 4.8 we have

$$
\begin{aligned}
\mathbb{E}\left[\mid f\left(\widetilde{X}_{N}^{\mathrm{f}}\right)\right. & \left.-\left.f\left(\widehat{X}_{N}^{\mathrm{f}}\right)\right|^{q\left(1-\delta^{\prime \prime}\right)}\right] \\
\leq & L_{f}^{q\left(1-\delta^{\prime \prime}\right)}\left(\mathbb{E}\left[\left.|1+c| \widetilde{X}_{N}^{\mathrm{f}}\right|^{r}+\left.c\left|\widehat{X}_{N}^{\mathrm{f}}\right|^{r}\right|^{q\left(1-\delta^{\prime \prime}\right) / \delta^{\prime \prime}}\right]\right)^{\delta^{\prime \prime}}\left(\mathbb{E}\left[\left|\widetilde{X}_{N}^{\mathrm{f}}-\widehat{X}_{N}^{\mathrm{f}}\right|^{q}\right]\right)^{1-\delta^{\prime \prime}} \\
& \prec\left(\mathbb{E}\left[|\widetilde{Z}-Z|^{q}\right]\right)^{1-\delta^{\prime \prime}}
\end{aligned}
$$




$$
\Longrightarrow \quad\left(\mathbb{E}\left[\left|f\left(\widetilde{X}_{N}^{\mathrm{f}}\right)-f\left(\widehat{X}_{N}^{\mathrm{f}}\right)\right|^{2 /\left(2 / q+\delta^{\prime}\right)}\right]\right)^{2 / q+\delta^{\prime}} \prec\left(\mathbb{E}\left[|\widetilde{Z}-Z|^{q}\right]\right)^{2 / q} .
$$

There is a similar bound for $\mathbb{E}\left[\left|f\left(\widetilde{X}_{N}^{\mathrm{c}}\right)-f\left(\widehat{X}_{N}^{\mathrm{c}}\right)\right|^{q\left(1-\delta^{\prime \prime}\right)}\right]$ and hence we have the result that $\mathbb{E}\left[(\Delta f)^{2} \mathbf{1}_{A \cup B}\right] \prec h^{(1-\delta) / 2-1 / q}\left(\mathbb{E}\left[|\widetilde{Z}-Z|^{q}\right]\right)^{2 / q}$ when $h^{1 / 2} \geq\left(\mathbb{E}\left[|\widetilde{Z}-Z|^{q}\right]\right)^{1 /(q+1)}$.

Combining the bounds from cases A and B with (9), we obtain the desired final result.

\section{Numerical results}

Our numerical tests are for the simplest possible example of Geometric Brownian Motion,

$$
\mathrm{d} X_{t}=\mu X_{t} \mathrm{~d} t+\sigma X_{t} \mathrm{~d} W_{t} .
$$

In our simulations we take $\mu=0.05, \sigma=0.2, T=1$, and $X_{0}=1$. The coarsest level $\ell=0$ uses a single time step, and higher levels use $4^{\ell}$ timesteps on level $\ell$ so that $h_{\ell}=2^{-2 l}$.

For the Normal random variables we use the approximations discussed in section 2 :

1. the quantised piecewise constant approximation using 1024 intervals;

2. the piecewise linear approximation on 16 dyadic intervals on $(0,1 / 2)$;

3. a degree 7 polynomial approximation.

Note that the values of $\mathbb{E}\left[|\widetilde{Z}-Z|^{2}\right]$ for these are $1.5 \times 10^{-4}, 4 \times 10^{-5}$ and $2.6 \times 10^{-3}$, respectively.

Figure 2 presents results for all three approximations for two different output functions, $f(x) \equiv x$ and $f(x) \equiv \max (x-1,0)$. In all cases the variances for $\mathbb{V}\left[\widetilde{P}_{\ell}-\widetilde{P}_{\ell-1}\right]$ using the approximate Normals are visually indistinguishable from $\mathbb{V}\left[\widehat{P}_{\ell}-\widehat{P}_{\ell-1}\right]$ which is the line labelled as "baseline"; the other three lines are the variances $\mathbb{V}\left[\left(\widehat{P}_{\ell}-\widehat{P}_{\ell-1}\right)-\left(\widetilde{P}_{\ell}-\widetilde{P}_{\ell-1}\right)\right]$ for the three approximations.

For the first case, $f(x) \equiv x$, by choosing $q$ close to 2 , Lemma 4.10 gives

$$
\widetilde{V}_{\ell} \equiv \mathbb{V}\left[\left(\widehat{P}_{\ell}-\widehat{P}_{\ell-1}\right)-\left(\widetilde{P}_{\ell}-\widetilde{P}_{\ell-1}\right)\right] \approx O\left(2^{-2 \ell} \mathbb{E}\left[|\widetilde{Z}-Z|^{2}\right]\right)
$$

The numerical results appear to be consistent with this, with $\widetilde{V}_{\ell}$ decreasing with level approximately proportional to $2^{-2 \ell}$, as indicated by the reference line which is proportional to $2^{-2 \ell}$. For a fixed level $\ell$, the variation in $\widetilde{V}_{\ell}$ between the three different approximations is roughly proportional to $\mathbb{E}\left[|\widetilde{Z}-Z|^{2}\right]$, with the piecewise linear approximation on dyadic intervals being the most accurate and hence giving the smallest values for $\widetilde{V}_{\ell}$, and the polynomial approximation being much less accurate leading to larger values for $\widetilde{V}_{\ell}$.

For the second case, $f(x) \equiv \max (x-1,0)$, choosing $\delta$ close to zero, Lemma 4.11 gives

$$
\widetilde{V}_{\ell} \approx O\left(\min \left\{2^{-2 \ell} \mathbb{E}\left[|\widetilde{Z}-Z|^{q}\right]^{1 /(q+1)}, 2^{-(1-2 / q) \ell} \mathbb{E}\left[|\widetilde{Z}-Z|^{q}\right]^{2 / q}\right\}\right),
$$

for any $q>2$, whereas the earlier heuristic analysis suggested

$$
\widetilde{V}_{\ell} \approx O\left(\min \left\{2^{-2 \ell} \mathbb{E}\left[|\widetilde{Z}-Z|^{2}\right]^{1 / 2}, 2^{-\ell} \mathbb{E}\left[|\widetilde{Z}-Z|^{2}\right]\right\}\right) .
$$

The numerical results are plotted with reference lines proportional to $2^{-\ell}$ and $2^{-2 \ell}$. The results do show a slight change in the slope reflecting the switch from $O\left(2^{-\ell}\right)$ to $O\left(2^{-2 \ell}\right)$ in the analysis. 

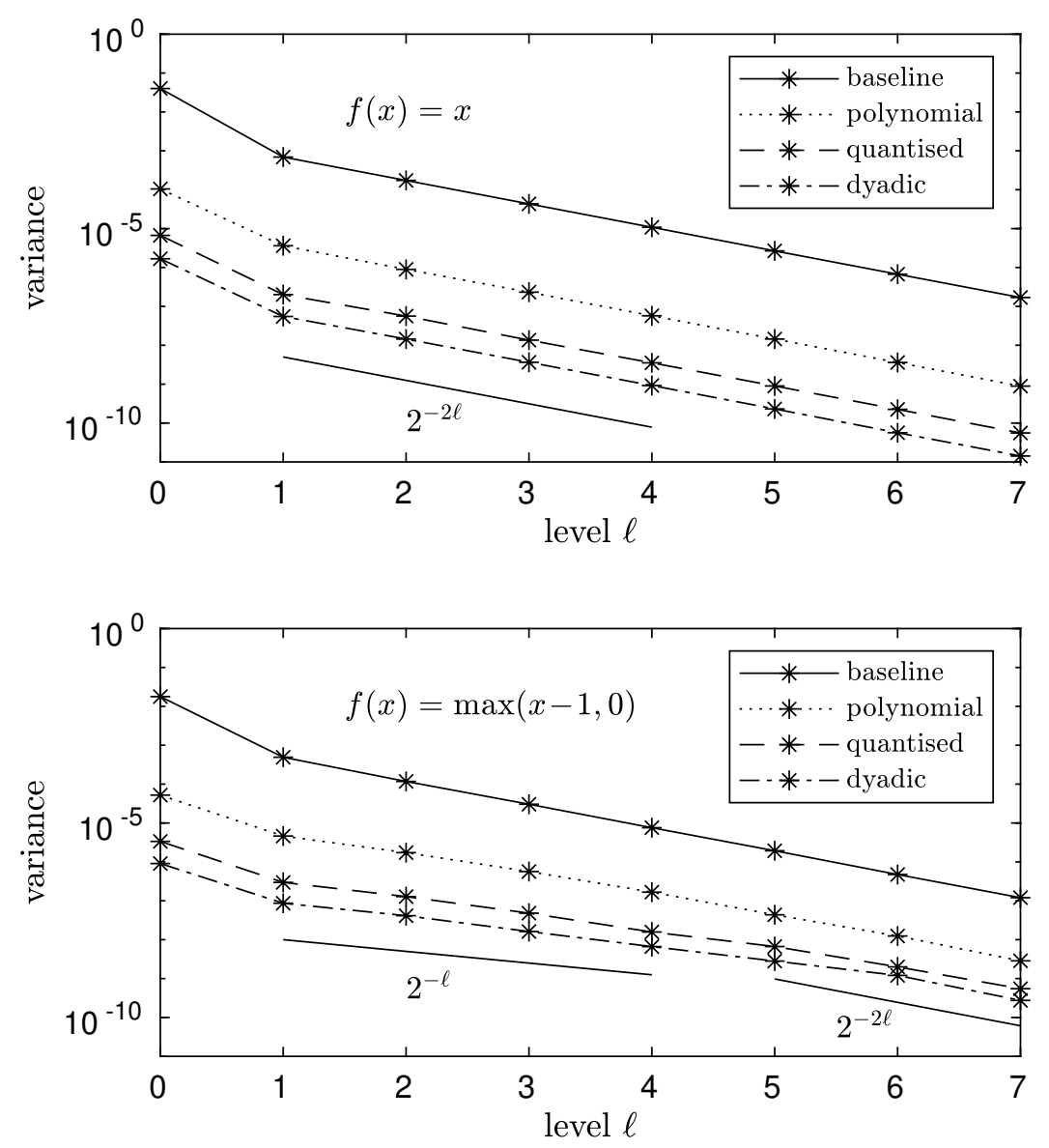

Figure 2: MLMC variances for two different output functions, with reference lines proportional to $2^{-2 \ell}$ and $2^{-\ell}$.

Regarding the overall computational efficiency, as discussed in section 3.2, the CPU implementations using the quantised and dyadic approximations are approximately 7 times more efficient, so $\widetilde{C}_{\ell} / C_{\ell} \approx 1 / 7$. The quantity $\sqrt{\left(C_{\ell} / \widetilde{C}_{\ell}+1\right) \widetilde{V}_{\ell} / V_{\ell}}$ is approximately 0.026 and 0.052 for the output function $f(x)=x$, using the dyadic and quantised approximations, respectively, and 0.14 and 0.19 for the output function $f(x)=\max (x-1,0)$, using the dyadic and quantised approximations. Therefore, in all four cases the total cost is reduced by a factor which is close to $\widetilde{C}_{\ell} / C_{\ell}$.

\section{Conclusions and future work}

In this paper we have presented a general nested multilevel Monte Carlo framework which employs approximate random variables which can be sampled much more efficiently than the true distribution. As a specific example, we investigated the use of approximate Normal random variables for an Euler-Maruyama discretisation of a scalar SDE. A detailed error analysis bounds the variance of the differences in the SDE path approximations as a function of the error in the approximate inverse Normal distribution. This analysis is supported by numerical results for the simplest possible case of Geometric Brownian Motion.

There are two directions in which we plan to extend this research. This first is to 
investigate approximations of other distributions. Two are of particular interest; one is the Poisson distribution, which is important for continuous-time Markov processes $[6,7]$ and is simulated using the inverse Normal CDF [30], and the other is the non-central $\chi^{2}-$ distribution which is important for simulating the Cox-Ingersoll-Ross (CIR) process which is used extensively in computational finance. In both cases, the computational savings may be greater, but it may prove to be very difficult to carry out a detailed numerical analysis of the resulting MLMC variances.

The second direction is to use reduced precision computer arithmetic in performing the calculations of $\widetilde{X}_{n}$, further reducing the cost of the approximate calculations. This builds on prior research by others, implementing MLMC methods on FPGAs (field-programmable gate arrays) [31, 32]. The rounding error effect of finite precision arithmetic can be modelled as an additional random error at each timestep; it is expected that on the coarsest levels with few timesteps this additional error will be small, but on the finest levels it may become significant and so perhaps such levels should be computed using single precision.

\section{Acknowledgements}

This publication is based on work supported by both the ICONIC EPSRC Programme Grant (EP/P020720/1) and the EPSRC Centre for Doctoral Training in Industrially Focused Mathematical Modelling (EP/L015803/1) in collaboration with Arm Ltd.

\section{References}

[1] S. Heinrich. Monte Carlo complexity of global solution of integral equations. Journal of Complexity, 14(2):151-175, 1998.

[2] M.B. Giles. Multilevel Monte Carlo path simulation. Operations Research, 56(3):607$617,2008$.

[3] K.A. Cliffe, M.B. Giles, R. Scheichl, and A. Teckentrup. Multilevel Monte Carlo methods and applications to elliptic PDEs with random coefficients. Computing and Visualization in Science, 14(1):3-15, 2011.

[4] A. Barth, C. Schwab, and N. Zollinger. Multi-level Monte Carlo finite element method for elliptic PDEs with stochastic coefficients. Numerische Mathematik, 119(1):123-161, 2011.

[5] M.B. Giles and C. Reisinger. Stochastic finite differences and multilevel Monte Carlo for a class of SPDEs in finance. SIAM Journal of Financial Mathematics, 3(1):572-592, 2012 .

[6] D. Anderson and D.J. Higham. Multi-level Monte Carlo for continuous time Markov chains, with applications in biochemical kinetics. SIAM Multiscale Modelling and Simulation, 10(1):146-179, 2012.

[7] D. Anderson, D.J. Higham, and Y. Sun. Complexity of multilevel Monte Carlo tauleaping. SIAM Journal of Numerical Analysis, 52(6):3106-3127, 2014.

[8] V.H. Hoang, C. Schwab, and A.M. Stuart. Complexity analysis of accelerated MCMC methods for Bayesian inversion. Inverse Problems, 29(8), 2013. 
[9] R. Scheichl, A. Stuart, and A. Teckentrup. Quasi-Monte Carlo and multilevel Monte Carlo methods for computing posterior expectations in elliptic inverse problems. SIAM/ASA Journal on Uncertainty Quantification, 5(1):466-492, 2017.

[10] K. Bujok, B. Hambly, and C. Reisinger. Multilevel simulation of functionals of Bernoulli random variables with application to basket credit derivatives. Methodology and Computing in Applied Probability, 17(3):579-604, 2015.

[11] M.B. Giles and T. Goda. Decision-making under uncertainty: using MLMC for efficient estimation of EVPPI. Statistics and Computing, 29(4):739-751, 2019.

[12] M.B. Giles, T. Nagapetyan, and K. Ritter. Multilevel Monte Carlo approximation of distribution functions and densities. SIAM/ASA Journal on Uncertainty Quantification, 3(1):267-295, 2015.

[13] C. Bierig and A. Chernov. Approximation of probability density functions by the multilevel Monte Carlo Maximum Entropy method. Journal of Computational Physics, 314:661-681, 2016.

[14] E. Ullmann and I. Papaioannou. Multilevel estimation of rare events. SIAM Journal on Uncertainty Quantification, 3(1):922-953, 2015.

[15] D. Elfverson, F. Hellman, and A. Målqvist. A multilevel Monte Carlo method for computing failure probabilities. SIAM/ASA Journal on Uncertainty Quantification, 4(1):312-330, 2016.

[16] M.B. Giles. Multilevel Monte Carlo methods. Acta Numerica, 24:259-328, 2015.

[17] M.B. Giles, M. Hefter, L. Mayer, and K. Ritter. Random bit quadrature and approximation of distributions on Hilbert spaces. Foundations of Computational Mathematics, 19(1):205-238, 2019.

[18] M.B. Giles, M. Hefter, L. Mayer, and K. Ritter. Random bit multilevel algorithms for stochastic differential equations. Journal of Complexity, 2019.

[19] E.H. Müller, R. Scheichl, and T. Shardlow. Improving multilevel Monte Carlo for stochastic differential equations with application to the Langevin equation. Royal Society Proceedings A, 471(2176), 2015.

[20] D. Belomestny and T. Nagapetyan. Multilevel path simulation for weak approximation schemes with application to Lévy-driven SDEs. Bernoulli, 23(2):927-950, 2017.

[21] M.B. Giles and O. Sheridan-Methven. Approximating inverse cumulative distribution functions to produce approximate random variables, 2020. arXiv 2012.09715.

[22] O. Sheridan-Methven. Nested multilevel Monte Carlo methods and a modified EulerMaruyama scheme utilising approximate Gaussian random variables suitable for vectorised hardware and low-precisions. PhD thesis, University of Oxford, 2020.

[23] N.S. Bakhvalov. Optimal convergence bounds for quadrature processes and integration methods of Monte Carlo type for classes of functions. Zh. Vychisl. Mat. i Mat. Fiz., 4(4, suppl.):5-63, 1964. 
[24] P. l'Ecuyer. Randomized quasi-Monte Carlo: An introduction for practitioners. In International Conference on Monte Carlo and Quasi-Monte Carlo Methods in Scientific Computing, pages 29-52. Springer, 2016.

[25] M.B. Giles, F.Y. Kuo, and I.H. Sloan. Combining sparse grids, multilevel MC and QMC for elliptic PDEs with random coefficients. In P.W. Glynn and A. Owen, editors, Monte Carlo and Quasi-Monte Carlo Methods 2016. Springer, 2018.

[26] A.-L. Haji-Ali, F. Nobile, and R. Tempone. Multi Index Monte Carlo: when sparsity meets sampling. Numerische Mathematik, 132(4):767-806, 2016.

[27] P.E. Kloeden and E. Platen. Numerical Solution of Stochastic Differential Equations. Springer, Berlin, 1992.

[28] D.L. Burkholder, B. Davis, and R.F. Gundy. Integral inequalities for convex functions of operators on martingales. In Proc. Sixth Berkeley Symposium Math. Statist. Prob., Vol II, pages 223-240. University of California Press, Berkeley, 1972.

[29] M.B. Giles and L. Szpruch. Antithetic multilevel Monte Carlo estimation for multidimensional SDEs without Lévy area simulation. Annals of Applied Probability, 24(4):1585-1620, 2014.

[30] M.B. Giles. Approximation of the inverse Poisson cumulative distribution function. ACM Transactions on Mathematical Software, 42(1), 2016.

[31] C. Brugger, C. de Schryver, N. Wehn, S. Omland, M. Hefter, K. Ritter, A. Kostiuk, and R. Korn. Mixed precision multilevel Monte Carlo on hybrid computing systems. In Proceedings of the Conference on Computational Intelligence for Financial Engineering and Economics. IEEE, 2014.

[32] S. Omland, M. Hefter, K. Ritter, C. Brugger, C. de Schryver, N. Wehn, and A. Kostiuk. Exploiting mixed-precision arithmetic in a multilevel Monte Carlo approach on FPGAs. In FPGA Based Accelerators for Financial Applications. Springer, 2015. 


\section{A Mean Value Theorem and a generalisation}

Lemma A.1 (Mean Value Theorem). If $f: \mathbb{R} \rightarrow \mathbb{R}$ is $C^{1}(\mathbb{R})$, then there exists $\xi$ which is a positively-weighted average of $x_{1}, x_{2}$ (i.e., $\xi=s x_{1}+(1-s) x_{2}$ for some $0<s<1$ ) such that

$$
f\left(x_{1}\right)-f\left(x_{2}\right)=\left(x_{1}-x_{2}\right) f^{\prime}(\xi) .
$$

Lemma A.2. If $f: \mathbb{R} \rightarrow \mathbb{R}$ is $C^{1}(\mathbb{R})$, and $f^{\prime}$ is Lipschitz continuous with Lipschitz constant $L_{f}^{\prime}$ then there exists $\xi$ which is a positively-weighted average of $x_{1}, x_{2}, x_{3}, x_{4}$ such that

$$
f\left(x_{1}\right)-f\left(x_{2}\right)-f\left(x_{3}\right)+f\left(x_{4}\right)=\left(x_{1}-x_{2}-x_{3}+x_{4}\right) f^{\prime}(\xi)+R,
$$

where

$$
|R| \leq \frac{1}{2} L_{f}^{\prime}\left(\left|x_{1}-x_{2}\right|+\left|x_{3}-x_{4}\right|\right)\left(\left|x_{1}-x_{3}\right|+\left|x_{2}-x_{4}\right|\right) .
$$

Proof. Without loss of generality, we can assume

$$
\left|x_{1}-x_{2}\right|+\left|x_{3}-x_{4}\right| \leq\left|x_{1}-x_{3}\right|+\left|x_{2}-x_{4}\right|,
$$

since otherwise we can just swap $x_{2}$ and $x_{3}$.

Now, using Lemma A.1 we get

$$
\begin{aligned}
& f\left(x_{1}\right)-f\left(x_{2}\right)=\left(x_{1}-x_{2}\right) f^{\prime}\left(\xi_{1}\right), \\
& f\left(x_{3}\right)-f\left(x_{4}\right)=\left(x_{3}-x_{4}\right) f^{\prime}\left(\xi_{2}\right),
\end{aligned}
$$

where $\xi_{1}$ and $\xi_{2}$ are positively-weighted averages of $x_{1}, x_{2}$ and $x_{3}, x_{4}$, respectively. Taking the difference gives

$$
f\left(x_{1}\right)-f\left(x_{2}\right)-f\left(x_{3}\right)+f\left(x_{4}\right)=\frac{1}{2}\left(x_{1}-x_{2}-x_{3}+x_{4}\right)\left(f^{\prime}\left(\xi_{1}\right)+f^{\prime}\left(\xi_{2}\right)\right)+R,
$$

where

$$
R=\frac{1}{2}\left(x_{1}-x_{2}+x_{3}-x_{4}\right)\left(f^{\prime}\left(\xi_{1}\right)-f^{\prime}\left(\xi_{2}\right)\right) .
$$

Since $f^{\prime}$ is continuous, there exists an $\xi$ which is a positively-weighted of $\xi_{1}$ and $\xi_{2}$, and hence of $x_{1}, x_{2}, x_{3}, x_{4}$ such that

$$
\frac{1}{2}\left(f^{\prime}\left(\xi_{1}\right)+f^{\prime}\left(\xi_{2}\right)\right)=f^{\prime}(\xi)
$$

Note that

$$
\xi_{1}-\xi_{2}=\left(\xi_{1}-\frac{1}{2}\left(x_{1}+x_{2}\right)\right)+\left(\xi_{2}-\frac{1}{2}\left(x_{3}+x_{4}\right)\right)+\left(\frac{1}{2}\left(x_{1}+x_{2}\right)-\frac{1}{2}\left(x_{3}+x_{4}\right)\right)
$$

and therefore, due to (10),

$$
\begin{aligned}
\left|\xi_{1}-\xi_{2}\right| & \leq \frac{1}{2}\left|x_{1}-x_{2}\right|+\frac{1}{2}\left|x_{3}-x_{4}\right|+\frac{1}{2}\left|x_{1}-x_{3}\right|+\frac{1}{2}\left|x_{2}-x_{4}\right| \\
& \leq\left|x_{1}-x_{3}\right|+\left|x_{2}-x_{4}\right|
\end{aligned}
$$

Hence, due to the Lipschitz property of $f^{\prime}$,

$$
|R| \leq \frac{1}{2} L_{f}^{\prime}\left(\left|x_{1}-x_{2}\right|+\left|x_{3}-x_{4}\right|\right)\left(\left|x_{1}-x_{3}\right|+\left|x_{2}-x_{4}\right|\right) .
$$

